\title{
A diffused interface with the advection term in a Sobolev space
}

\author{
Yoshihiro Tonegawa $^{1} \cdot$ Yuki Tsukamoto $^{1}$
}

Received: 31 March 2019 / Accepted: 31 August 2020 / Published online: 9 October 2020

(c) The Author(s) 2020

\begin{abstract}
We study the asymptotic limit of diffused surface energy in the van der Waals-Cahn-Hillard theory when an advection term is added and the energy is uniformly bounded. We prove that the limit interface is an integral varifold and the generalized mean curvature vector is determined by the advection term. As the application, a prescribed mean curvature problem is solved using the min-max method.
\end{abstract}

Mathematics Subject Classification 49Q20

\section{Introduction}

The object of study in this paper is the energy functional appearing in the van der WaalsCahn-Hillard theory [4,7],

$$
E_{\varepsilon}(u)=\int_{\Omega} \frac{\varepsilon|\nabla u|^{2}}{2}+\frac{W(u)}{\varepsilon},
$$

where $u: \Omega \subset \mathbb{R}^{n} \rightarrow \mathbb{R}(n \geq 2)$ is the normalized density distribution of two phases of a material, $|\nabla u|^{2}=\sum_{k=1}^{n}\left(\partial u / \partial x_{k}\right)^{2}$ and $W: \mathbb{R} \rightarrow[0, \infty)$ is a double-well potential with two global minima at \pm 1 . In the thermodynamic context, $W$ corresponds to the Helmholtz free energy density and the typical example is $W(u)=\left(1-u^{2}\right)^{2}$. When the positive parameter $\varepsilon$ is small relative to the size of the domain $\Omega$ and $E_{\varepsilon}(u)$ is bounded, it is expected that $u$ is close to +1 or -1 on most of $\Omega$ while a spatial change between \pm 1 occurs within a hypersurface-like region of $O(\varepsilon)$ thickness which we may call the diffused interface of $u$. In this case, the quantity $E_{\varepsilon}(u)$ is expected to be proportional to the surface area of the diffused

Communicated by A. Neves.

The first author is partially supported by JSPS KAKENHI Grant Numbers (A) 25247008 and (S) 26220702.

$凶$ Yoshihiro Tonegawa

tonegawa@math.titech.ac.jp

Yuki Tsukamoto

tsukamoto.y.ag@m.titech.ac.jp

1 Department of Mathematics, Tokyo Institute of Technology, Tokyo 152-8551, Japan 
interface. Due to the importance of the surface area in calculus of variations, it is interesting to investigate the validity of such expectation and other salient properties of $E_{\varepsilon}$.

In this direction, there have been a number of works studying the asymptotic behavior of $E_{\varepsilon}$ as $\varepsilon \rightarrow 0+$ under various assumptions. For the energy minimizers with appropriate side conditions, it is well-known that it $\Gamma$-converges to the area functional of the limit interface $[10,12-14,20]$. On the other hand, due in part to the non-convex nature of the functional, there may exist multiple and even infinite number of critical points of $E_{\varepsilon}$ different from the energy minimizers. For general critical points, Hutchinson and the first author [8] proved that the limit is an integral stationary varifold [1]. For general stable critical points, the first author and Wickramasekera [26] proved that the limit is an embedded real-analytic minimal hypersurface except for a closed singular set of codimension seven. More recently, Guaraco [6] showed that a uniform Morse index bound is sufficient to conclude the same regularity for $n \geq 3$ and gave a new proof of Almgren-Pitts theorem [16] as the application. The new proof significantly simplifies the existence part of the proof even though one needs to use Wickramasekera's hard regularity theorem [27].

While the investigations on the critical points of $E_{\varepsilon}$ have direct links to the minimal surface theory as above, more generally, it turned out that suitable controls of the first variation of $E_{\varepsilon}$ guarantee the analogous good asymptotic behaviors. For example, under the assumption that

$$
\liminf _{\varepsilon \rightarrow 0+}\left(E_{\varepsilon}\left(u_{\varepsilon}\right)+\left\|f_{\varepsilon}\right\|_{W^{1, p}(\Omega)}\right)<\infty
$$

with $f_{\varepsilon}:=-\varepsilon \Delta u_{\varepsilon}+W^{\prime}\left(u_{\varepsilon}\right) / \varepsilon$ and $p>n / 2$, the first author [22,25] proved that the limit interface is an integral varifold whose generalized mean curvature belongs to $L^{q}$ ( $q=$ $p(n-1) /(n-p)>n-1)$ with respect to the surface measure. Here $W^{1, p}(\Omega):=\{u \in$ $\left.L^{p}(\Omega): \nabla u \in L^{p}(\Omega)\right\}$. The mean curvature of the limit interface is characterized by the weak $W^{1, p}$ limit of $f_{\varepsilon}[18]$. Another example concerns one of De Giorgi's conjectures. Under the assumption that (with $f_{\varepsilon}$ as above)

$$
\liminf _{\varepsilon \rightarrow 0+}\left(E_{\varepsilon}\left(u_{\varepsilon}\right)+\varepsilon^{-1}\left\|f_{\varepsilon}\right\|_{L^{2}(\Omega)}^{2}\right)<\infty
$$

and $n=2,3$, Röger-Schätzle [17] (independently [15] for the case of $n=2$ ) proved the similar result. In this case, the limit interface has an $L^{2}$ generalized mean curvature.

In this paper, along the line of research described above, we investigate the asymptotic behavior of $u_{\varepsilon}$ satisfying

$$
-\varepsilon \Delta u_{\varepsilon}+\frac{W^{\prime}\left(u_{\varepsilon}\right)}{\varepsilon}=\varepsilon v_{\varepsilon} \cdot \nabla u_{\varepsilon},
$$

where $v_{\varepsilon}$ is considered here as a given vector field and we assume that

$$
\liminf _{\varepsilon \rightarrow 0+}\left(E_{\varepsilon}\left(u_{\varepsilon}\right)+\left\|v_{\varepsilon}\right\|_{W^{1, p}(\Omega)}\right)<\infty
$$

and $p>n / 2$. The problem is related to (parabolic) Allen-Cahn-type equations studied in $[11,21]$, for example. It is also natural to investigate the effect of advection term as $\varepsilon \rightarrow 0+$. We prove the analogous result Theorem 2.1 to [22,25], namely, the limit is an integral varifold with $L^{q}$ (the same as above) generalized mean curvature which is characterized by the weak $W^{1, p}$ limit of $v_{\varepsilon}$. Using this result, we give some existence theorem for a vectorial prescribed mean curvature problem, as described in Theorem 2.2. Despite the simplicity of the problem, this is the first existence result in the setting of the min-max method, with minimal regularity assumptions on the prescribed vector field. As for the existence problem for scalar constant 
or prescribed mean curvature using a min-max approach along the lines of Almgren-Pitts [16], we mention papers by Zhou and Zhu [28,29].

As for the proof, just as in the case of $[8,22,25]$, the key point is to prove a certain monotonicity-type formula which is the essential tool in the setting of Geometric Measure Theory. We wish to treat $\varepsilon v_{\varepsilon} \cdot \nabla u_{\varepsilon}$ as a perturbative term, and to do so, we need to control a certain "trace" norm of $v_{\varepsilon}$ on diffused interface. If an $\varepsilon$-independent upper density ratio estimate of diffused surface measure is available, then we can control $\varepsilon v_{\varepsilon} \cdot \nabla u_{\varepsilon}$ by the $W^{1, p}$ norm of $v_{\varepsilon}$. For this purpose, we establish the key estimate, Theorem 3.8, which gives a local uniform upper density ratio estimate. Once this part is done, the rest proceeds just like [25] with minor modifications.

The paper is organized as follows. In Sect. 2 we state our assumptions and explain the main results. Section 3 contains the main estimates which ultimately give a monotonicitytype formula, Theorem 3.9. In Sect. 4, we prove the main theorem by modifying the proof in $[22,25]$, and in Sect. 5, we give some concluding remarks.

\section{Assumptions and main results}

We use the notation that $U_{r}(a):=\left\{x \in \mathbb{R}^{n}:|x-a|<r\right\}, B_{r}(a):=\left\{x \in \mathbb{R}^{n}:|x-a| \leq r\right\}$, $U_{r}:=U_{r}(0)$ and $B_{r}:=B_{r}(0)$.

\subsection{Assumptions}

Throughout the paper, we assume that:

(a) The function $W: \mathbb{R} \rightarrow[0, \infty)$ is $C^{3}$ and has two strict minima $W( \pm 1)=W^{\prime}( \pm 1)=0$.

(b) For some $\gamma \in(-1,1), W^{\prime}>0$ on $(-1, \gamma)$ and $W^{\prime}<0$ on $(\gamma, 1)$.

(c) For some $\alpha \in(0,1)$ and $\kappa>0, W^{\prime \prime}(x) \geq \kappa$ for all $|x| \geq \alpha$.

Let $\Omega \subset \mathbb{R}^{n}$ be a bounded domain. We assume that we are given $W^{1,2}(\Omega)$ functions $\left\{u_{i}\right\}_{i=1}^{\infty}$, $W^{1, p}\left(\Omega ; \mathbb{R}^{n}\right)$ vector fields $\left\{v_{i}\right\}_{i=1}^{\infty}$ and positive constants $\left\{\varepsilon_{i}\right\}_{i=1}^{\infty}$ satisfying

$$
-\varepsilon_{i} \Delta u_{i}+\frac{W^{\prime}\left(u_{i}\right)}{\varepsilon_{i}}=\varepsilon_{i} v_{i} \cdot \nabla u_{i}
$$

weakly on $\Omega$ for each $i \in \mathbb{N}$. In addition, assume that

$$
\lim _{i \rightarrow \infty} \varepsilon_{i}=0, \quad \frac{n}{2}<p<n
$$

and that there exist constants $c_{0}, E_{0}$ and $\lambda_{0}$ such that, for all $i \in \mathbb{N}$, we have:

$$
\begin{aligned}
& \left\|u_{i}\right\|_{L^{\infty}(\Omega)} \leq c_{0}, \\
& \int_{\Omega}\left(\frac{\varepsilon_{i}\left|\nabla u_{i}\right|^{2}}{2}+\frac{W\left(u_{i}\right)}{\varepsilon_{i}}\right) \leq E_{0}, \\
& \left\|v_{i}\right\|_{L^{\frac{n p}{n-p}}(\Omega)}+\left\|\nabla v_{i}\right\|_{L^{p}(\Omega)} \leq \lambda_{0} .
\end{aligned}
$$

The condition (2.3) is not essential and can be often derived from the PDE or the proof of existence. Here we assume (2.3) for simplicity. Next, define

$$
\Phi(s):=\int_{-1}^{s} \sqrt{W(t) / 2} d t, \quad w_{i}(x):=\Phi\left(u_{i}(x)\right) .
$$


By the Cauchy-Schwarz inequality and (2.4), we obtain

$$
\int_{\Omega}\left|\nabla w_{i}\right| \leq \frac{1}{2} \int_{\Omega}\left(\frac{\varepsilon_{i}\left|\nabla u_{i}\right|^{2}}{2}+\frac{W\left(u_{i}\right)}{\varepsilon_{i}}\right) \leq \frac{1}{2} E_{0} .
$$

Hence, by the compactness theorem for $B V$ functions [30, Corollary 5.3.4], there exist a converging subsequence (which we denote by the same notation) $\left\{w_{i}\right\}$ in the $L^{1}$ norm and the limit BV function $w$. Define

$$
u(x):=\Phi^{-1}(w(x)) .
$$

where $\Phi^{-1}$ is the inverse function of $\Phi$. It follows that $u_{i}$ converges to $u$ a.e. on $\Omega$. By Fatou's Lemma and (2.4), we have

$$
\int_{\Omega} W(u)=\int_{\Omega} \lim _{i \rightarrow \infty} W\left(u_{i}\right) \leq \liminf _{i \rightarrow \infty} \int_{\Omega} W\left(u_{i}\right)=0 .
$$

This shows that $u= \pm 1$ a.e. on $\Omega$ and $u$ is a $B V$ function. For simplicity we write $\partial^{*}\{u=1\}$ as the reduced boundary [30] of $\{u=1\}$ and $\left\|\partial^{*}\{u=1\}\right\|$ as the boundary measure.

\subsection{The associated varifolds}

We associate to each solution of (1.2) a varifold in a natural way in the following. We refer to $[1,19]$ for a comprehensive treatment of varifolds.

Let $\mathbf{G}(n, n-1)$ be the Grassmannian, i.e. the space of unoriented $(n-1)$-dimensional subspaces in $\mathbb{R}^{n}$. We also regard $S \in \mathbf{G}(n, n-1)$ as the $n \times n$ matrix representing the orthogonal projection of $\mathbb{R}^{n}$ onto $S$. For two given square-matrices $S_{1}$ and $S_{2}$, we write $S_{1} \cdot S_{2}:=\operatorname{trace}\left(S_{1}^{t} \circ S_{2}\right)$, where the upper-script $t$ indicates the transpose of the matrix and $\circ$ is the matrix multiplication. We say that $V$ is an $(n-1)$-dimensional varifold in $\Omega \subset \mathbb{R}^{n}$ if $V$ is a Radon measure on $\mathbf{G}_{n-1}(\Omega):=\Omega \times \mathbf{G}(n, n-1)$. Let $\mathbf{V}_{n-1}(\Omega)$ be the set of all $(n-1)$-dimensional varifolds in $\Omega$. Convergence in the varifold sense means convergence in the usual sense of measures. For $V \in \mathbf{V}_{n-1}(\Omega)$, we let $\|V\|$ be the weight measure of $V$. For $V \in \mathbf{V}_{n-1}(\Omega)$, we define the first variation of $V$ by

$$
\delta V(g):=\int_{\mathbf{G}_{n-1}(\Omega)} \nabla g(x) \cdot S d V(x, S)
$$

for any vector field $g \in C_{c}^{1}\left(\Omega ; \mathbb{R}^{n}\right)$. We let $\|\delta V\|$ be the total variation of $\delta V$. If $\|\delta V\|$ is absolutely continuous with respect to $\|V\|$, then the Radon-Nikodym derivative $\delta V /\|V\|$ exists as a vector-valued $\|V\|$ measurable function. In this case, we define the generalized mean curvature vector of $V$ by $-\delta V /\|V\|$ and we use the notation $H_{V}$.

We associate to each function $u_{i}$ a varifold $V_{i}$ as follows. First, we define a Radon measure $\mu_{i}$ on $\Omega$ by

$$
d \mu_{i}:=\frac{1}{\sigma}\left(\frac{\varepsilon_{i}\left|\nabla u_{i}\right|^{2}}{2}+\frac{W\left(u_{i}\right)}{\varepsilon_{i}}\right) d \mathcal{L}^{n},
$$

where $\mathcal{L}^{n}$ is the $n$-dimensional Lebesgue measure and $\sigma:=\int_{-1}^{1} \sqrt{2 W(s)} d s$. Define $V_{i} \in$ $\mathbf{V}_{n-1}(\Omega)$ by

$$
V_{i}(\phi):=\int_{\left\{\left|\nabla u_{i}\right| \neq 0\right\}} \phi\left(x, I-\frac{\nabla u_{i}(x)}{\left|\nabla u_{i}(x)\right|} \otimes \frac{\nabla u_{i}(x)}{\left|\nabla u_{i}(x)\right|}\right) d \mu_{i}(x)
$$


for $\phi \in C_{c}\left(\mathbf{G}_{n-1}(\Omega)\right)$, where $I$ is the $n \times n$ identity matrix and $\otimes$ is the tensor product of the two vectors. Note that $I-\frac{\nabla u_{i}(x)}{\left|\nabla u_{i}(x)\right|} \otimes \frac{\nabla u_{i}(x)}{\left|\nabla u_{i}(x)\right|}$ represents the orthogonal projection to the $(n-1)$-dimensional subspace $\left\{a \in \mathbb{R}^{n}: a \cdot \nabla u_{i}(x)=0\right\}$. By definition, we have

$$
\left\|V_{i}\right\|=\mu_{i}\left\llcorner_{\left\{\left|\nabla u_{i}\right| \neq 0\right\}}\right.
$$

and by (2.6), we have

$$
\delta V_{i}(g)=\int_{\left\{\left|\nabla u_{i}\right| \neq 0\right\}} \nabla g \cdot\left(I-\frac{\nabla u_{i}}{\left|\nabla u_{i}\right|} \otimes \frac{\nabla u_{i}}{\left|\nabla u_{i}\right|}\right) d \mu_{i}
$$

for each $g \in C_{c}^{1}\left(\Omega, \mathbb{R}^{n}\right)$.

\subsection{Main Theorems}

With the above assumptions and notation, we show:

Theorem 2.1 Suppose that $u_{i}, v_{i}, \varepsilon_{i}$ satisfy (2.1)-(2.5) and let $V_{i}$ be the varifold associated with $u_{i}$ as in (2.8). On passing to a subsequence we can assume that

$$
v_{i} \rightarrow v \text { weakly in } W^{1, p}, u_{i} \rightarrow u \text { a.e., } V_{i} \rightarrow V \text { in the varifold sense. }
$$

Then we have the following properties.

(1) For each $\phi \in C_{c}(\Omega)$,

$$
\begin{aligned}
\frac{1}{2}\|V\|(\phi) & =\lim _{i \rightarrow \infty} \frac{1}{\sigma} \int_{\Omega} \frac{\varepsilon_{i}}{2}\left|\nabla u_{i}\right|^{2} \phi=\lim _{i \rightarrow \infty} \frac{1}{\sigma} \int_{\Omega} \frac{W\left(u_{i}\right)}{\varepsilon_{i}} \phi \\
& =\lim _{i \rightarrow \infty} \frac{1}{\sigma} \int_{\Omega}\left|\nabla w_{i}\right| \phi .
\end{aligned}
$$

(2) spt $\left\|\partial^{*}\{u=1\}\right\| \subset$ spt $\|V\|$ and $\left\{u_{i}\right\}$ converges locally uniformly to \pm 1 on $\Omega \backslash$ spt $\|V\|$.

(3) For each $0<b<1,\left\{\left|u_{i}\right| \leq 1-b\right\}$ locally converges to spt $\|V\|$ in the Hausdorff distance sense in $\Omega$.

(4) $V$ is an integral varifold and the density $\theta(x)$ of $V$ satisfies

$$
\theta(x)= \begin{cases}\text { odd } & \mathcal{H}^{n-1} \text { a.e. } x \in \partial^{*}\{u=1\}, \\ \text { even } & \mathcal{H}^{n-1} \text { a.e. } x \in \operatorname{spt}\|V\| \backslash \partial^{*}\{u=1\},\end{cases}
$$

(5) the generalized mean curvature vector $H_{V}$ of $V$ is given by

$$
H_{V}(x)=\left(T_{x} \operatorname{spt}\|V\|\right)^{\perp}(v(x)),
$$

for $\|V\|$ a.e. $x \in \Omega$.

(6) For $\tilde{\Omega} \subset \subset \Omega$, there exists a constant $\lambda_{1}$ depending only on $c_{0}, \lambda, \lambda_{0}, n, p, W, E_{0}$ and $\operatorname{dist}(\tilde{\Omega}, \partial \Omega)$ such that

$$
\int_{\tilde{\Omega}}\left|H_{V}(x)\right|^{\frac{p(n-1)}{n-p}} d\|V\|(x) \leq \int_{\tilde{\Omega}}|v(x)|^{\frac{p(n-1)}{n-p}} d\|V\|(x) \leq \lambda_{1} .
$$

Note that $\frac{p(n-1)}{n-p}>n-1$ due to (2.2). 
Since $V$ is integral and the generalized mean curvature vector is in the stated class, $V$ satisfies various good properties described in [19, Section 17]. In particular, spt $\|V\|$ is a closed countably $(n-1)$-rectifiable set (see $[19,17.9(1)])$, and writing $\Gamma:=$ spt $\|V\|$, for any $\phi \in C_{c}\left(\mathbf{G}_{n-1}(\Omega)\right)$,

$$
\int_{\mathbf{G}_{n-1}(\Omega)} \phi(x, S) d V(x, S)=\int_{\Gamma} \phi\left(x, T_{x} \Gamma\right) \theta(x) d \mathcal{H}^{n-1}(x) .
$$

Here, $T_{x} \Gamma \in \mathbf{G}(n, n-1)$ is the approximate tangent space of $\Gamma$ at $x$ which exists $\mathcal{H}^{n-1}$ a.e. $x \in \Gamma$. With this notation, (5) implies that $H_{V}(x)=\left(T_{x} \Gamma\right)^{\perp}(v(x))$ for $\mathcal{H}^{n-1}$ a.e. $x \in$ $\Gamma$, i.e., the generalized mean curvature vector of $V$ coincides with the projection of $v$ to the orthogonal subspace $\left(T_{x} \Gamma\right)^{\perp}$ for $\mathcal{H}^{n-1}$ a.e. $x \in \Gamma$. We emphasize the difference of characterization of the mean curvature vector from [25], where the similar equality holds only on the reduced boundary of $\{u=1\}$, while the equality here holds on the whole support of $\|V\|$ including on the "hidden boundary" $\Gamma \backslash \partial^{*}\{u=1\}$. If we additionally assume that $\theta=1$ for $\mathcal{H}^{n-1}$ a.e. $x \in \Gamma$, then because of the integrability of $H_{V}$ and the Allard regularity theorem [1], except for a closed $\mathcal{H}^{n-1}$-null set, $\Gamma$ is locally a $C^{1,2-\frac{n}{p}}$ hypersurface. Without the assumption $\theta=1$, we can still conclude that spt $\|V\|$ is $C^{1,2-\frac{n}{p}}$ hypersurface on a dense open set of spt $\|V\|$, even though we do not know if the complement is $\mathcal{H}^{n-1}$-null or not.

\subsection{A vectorial prescribed mean curvature problem}

As an application ${ }^{1}$ of Theorem 2.1 with suitable modifications, we prove the following:

Theorem 2.2 Let $(M, g)$ be a smooth compact $n$-dimensional Riemannian manifold and let $\rho \in W^{2, p}(M)$ be a given function, where $p>\frac{n}{2}$. Then, there exists a non-zero integral varifold $V$ in $M$ such that

$$
H_{V}(x)=\left(T_{x} \text { spt }\|V\|\right)^{\perp}(\nabla \rho(x))
$$

for $\|V\|$ a.e. $x \in M$.

Proof We may assume $p<n$. Consider the following functional for $\varepsilon>0$ and $u \in$ $W^{1,2}(M)$ :

$$
F_{\varepsilon}(u):=\int_{M}\left(\frac{\varepsilon|\nabla u|^{2}}{2}+\frac{W(u)}{\varepsilon}\right) \exp (\rho) d \omega_{g} .
$$

By the Sobolev embedding, $\rho \in C^{0,2-\frac{n}{p}}(M)$ and thus $0<\exp (\min \rho) \leq \exp (\rho) \leq$ $\exp (\max \rho)<\infty$. By considering the path space in $W^{1,2}(M)$ connecting $u \equiv 1$ and $u \equiv-1$, the standard min-max method gives a non-trivial critical point $u_{\varepsilon}$ for each $\varepsilon>0$, with uniform strictly positive lower and upper bounds of $F_{\varepsilon}\left(u_{\varepsilon}\right)$. The critical point satisfies $(2.1)$ with $v=\nabla \rho$ and $\left|u_{\varepsilon}\right| \leq 1$, with an appropriate modification of the equation. Take a sequence $\varepsilon_{i} \rightarrow 0+$ and a corresponding min-max critical points $u_{i}$. Then the sequence $u_{i}, \nabla \rho, \varepsilon_{i}$ satisfy all the assumptions of Theorem 2.1 with a small error terms coming from the metric of $M$ (see Guaraco's work [6] for an adaptation of the results to Riemannian manifolds in the case $\left.v_{\varepsilon}=0\right)$. The limit varifold $V$ thus has the desired property.

For more remarks on the main results, see Sect. 5.

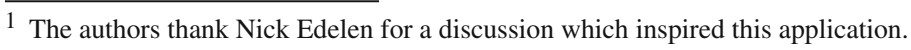




\section{The estimate for the upper density ratio}

In this section, we prove Theorem 3.8-3.10, which give $\varepsilon$-independent estimates of the upper and lower density ratios of the energy. Throughout this section, we drop the index $i$ and set $\Omega=U_{1}=\{|x|<1\}$ since the result is local. Assume $u \in W^{1,2}\left(U_{1}\right)$ and $v \in W^{1, p}\left(U_{1} ; \mathbb{R}^{n}\right)$ satisfy (2.1) with a positive $\varepsilon$ and (2.3)-(2.5) are satisfied for a given set of $c_{0}, E_{0}, \lambda_{0}$. The exponent $p$ satisfies (2.2). We first derive two preliminary properties for $u$, Lemmas 3.1 and 3.2 .

Lemma 3.1 There exists $c_{1}>0$ depending only on $c_{0}, \lambda_{0}, n, p$ and $W$ such that

$$
\sup _{x \in U_{1-\varepsilon}} \varepsilon|\nabla u(x)| \leq c_{1}
$$

and

$$
\sup _{x, x^{\prime} \in U_{1-\varepsilon}} \varepsilon^{3-\frac{n}{p}} \frac{\left|\nabla u(x)-\nabla u\left(x^{\prime}\right)\right|}{\left|x-x^{\prime}\right|^{2-\frac{n}{p}}} \leq c_{1}
$$

for $0<\varepsilon<1 / 2$. If $\varepsilon \geq 1 / 2$, then we have for any $0<s<1$

$$
\sup _{x \in U_{s}}|\nabla u(x)| \leq c_{1}
$$

where $c_{1}$ depends additionally on $s$. In both cases, we have $u \in W_{l o c}^{3, p}\left(U_{1}\right)$.

Proof Consider the case $0<\varepsilon<1 / 2$. Define $\tilde{u}(x):=u(\varepsilon x)$ and $\tilde{v}(x):=\varepsilon v(\varepsilon x)$ for $x \in U_{\varepsilon^{-1}}$. After this change of variables, we obtain from (2.1) that

$$
-\Delta \tilde{u}+W^{\prime}(\tilde{u})=\tilde{v} \cdot \nabla \tilde{u} \text { weakly on } U_{\varepsilon^{-1}} .
$$

Under the change of variables, we obtain from (2.5)

$$
\|\tilde{v}\|_{L^{\frac{n p}{n-p}}\left(U_{\varepsilon^{-1}}\right)}+\|\nabla \tilde{v}\|_{L^{p}\left(U_{\varepsilon^{-1}}\right)} \leq \lambda_{0} \varepsilon^{2-\frac{n}{p}}
$$

For any $U_{2}(x) \subset U_{\varepsilon^{-1}}$, let $\phi \in C_{c}^{1}\left(U_{2}(x)\right)$ be a function such that $0 \leq \phi \leq 1, \phi=1$ on $B_{1}(x)$ and $|\nabla \phi| \leq 4$ on $U_{2}(x)$. Use (3.4) with the test function $\tilde{u} \phi^{2}$. Using also (2.3), we obtain

$$
\begin{aligned}
\int|\nabla \tilde{u}|^{2} \phi^{2} & \leq c_{0} \int\left(2 \phi\left|\nabla \phi\left\|\nabla \tilde{u}|+| W^{\prime}\left|\phi^{2}+\right| \tilde{v}\right\| \nabla \tilde{u}\right| \phi^{2}\right) \\
& \leq \frac{1}{2} \int|\nabla \tilde{u}|^{2} \phi^{2}+\int\left(4 c_{0}^{2}|\nabla \phi|^{2}+c_{0}\left|W^{\prime}\right| \phi^{2}+c_{0}^{2}|\tilde{v}|^{2} \phi^{2}\right) .
\end{aligned}
$$

Since $\frac{n p}{n-p}>2,(3.5)$ and (3.6) give

$$
\sup _{B_{2}(x) \subset U_{\varepsilon^{-1}}} \int_{B_{1}(x)}|\nabla \tilde{u}|^{2} \leq c\left(c_{0}, \lambda_{0}, n, p, W\right) .
$$

We next note that the function $\tilde{u} \phi$ weakly satisfies the following equation:

$$
-\Delta(\tilde{u} \phi)=-\tilde{u} \Delta \phi-2 \nabla \phi \cdot \nabla \tilde{u}+\left(\tilde{v} \cdot \nabla \tilde{u}-W^{\prime}(\tilde{u})\right) \phi .
$$


Using the standard $L^{p}$ theory [5, Theorem 9.11] to (3.8), we may start a bootstrapping argument as follows. Staring with $q=2$, we have

$$
\begin{aligned}
\nabla \tilde{u} & \in L_{l o c}^{q} \Longrightarrow \tilde{v} \cdot \nabla \tilde{u} \in L_{l o c}^{\frac{n p q}{n p+q(n-p)}} \Longrightarrow \tilde{u} \in W_{l o c}^{2, \frac{n p q}{n p+q(n-p)}} \\
& \Longrightarrow \nabla \tilde{u} \in L_{l o c}^{\frac{n p q}{n p-q(2 p-n)}}
\end{aligned}
$$

with the corresponding estimates relating these norms. Note that the exponent of integrability of $\nabla \tilde{u}$ is raised from $q$ to $q \cdot \frac{n p}{n p-q(2 p-n)}$, with the factor strictly larger than one. Thus, in a finite number of bootstrapping, we obtain the $W_{l o c}^{2, s}$ (with $s>n$ ) estimate for $\tilde{u}$, and by the Sobolev inequality, the $L_{l o c}^{\infty}$ estimate for $\nabla \tilde{u}$. Again by the $L^{p}$ theory, we obtain the $W_{l o c}^{2, \frac{n p}{n-p}}$ estimate of $\tilde{u}$. In particular, by the Sobolev inequality, we obtain (3.1) and (3.2). Since the right-hand side of (3.8) is in $W_{l o c}^{1, p}$ (note that $\tilde{v} \cdot \nabla^{2} \tilde{u} \in L_{l o c}^{\frac{n p}{2(n-p)}}$ and $\frac{n p}{2(n-p)}>p$ by (2.2)), we have $\tilde{u} \in W_{\text {loc }}^{3, p}$ and the weak third-derivatives of $\tilde{u}$ exist. The case of $\varepsilon \geq 1 / 2$ does not require the change of variables as above and the proof is omitted.

Lemma 3.2 Given $0<s<1$, there exist constants $0<\varepsilon_{1}, \eta<1$ depending only on $c_{0}, \lambda_{0}, W, n, p$ and such that

$$
\sup _{x \in B_{s}}|u(x)| \leq 1+\varepsilon^{\eta}
$$

for $\varepsilon \leq \varepsilon_{1}$.

Proof Let $q=\frac{n p}{n-p}-1$ and $\phi \in C_{c}^{\infty}\left(B_{\frac{s+1}{2}}\right)$ with $\phi \geq 0$. Multiplying $(2.1)$ by $\left[(u-1)_{+}\right]^{q} \phi^{2}$, we have

$$
\begin{aligned}
& -\varepsilon \int q\left[(u-1)_{+}\right]^{q-1}|\nabla u|^{2} \phi^{2}+2\left[(u-1)_{+}\right]^{q} \phi \nabla \phi \cdot \nabla u \\
& =\int \frac{W^{\prime}}{\varepsilon}\left[(u-1)_{+}\right]^{q} \phi^{2}-\int \varepsilon \nabla u \cdot v\left[(u-1)_{+}\right]^{q} \phi^{2} .
\end{aligned}
$$

By $W^{\prime}(u) \geq \kappa(u-1)$ for $u \geq 1$ and (3.1), we obtain

$$
\begin{aligned}
& \frac{\kappa}{\varepsilon} \int\left[(u-1)_{+}\right]^{q+1} \phi^{2}+\int \varepsilon q\left[(u-1)_{+}\right]^{q-1}|\nabla u|^{2} \phi^{2} \\
& \leq 2 \varepsilon \int\left[(u-1)_{+}\right]^{q} \phi|\nabla \phi||\nabla u|+c_{1} \int|v|\left[(u-1)_{+}\right]^{q} \phi^{2} \\
& \leq \frac{q \varepsilon}{2} \int\left[(u-1)_{+}\right]^{q-1}|\nabla u|^{2} \phi^{2}+\frac{8 \varepsilon}{q} \int\left[(u-1)_{+}\right]^{q+1}|\nabla \phi|^{2} \\
& \quad+\frac{\kappa}{2 \varepsilon} \int\left[(u-1)_{+}\right]^{q+1} \phi^{2}+\frac{\varepsilon^{q} c\left(q, c_{1}\right)}{\kappa^{q}} \int|v|^{q+1} \phi^{2},
\end{aligned}
$$

which shows

$$
\frac{\kappa}{2 \varepsilon} \int\left[(u-1)_{+}\right]^{q+1} \phi^{2} \leq \frac{8 \varepsilon}{q} \int\left[(u-1)_{+}\right]^{q+1}|\nabla \phi|^{2}+\frac{\varepsilon^{q} c\left(q, c_{1}\right)}{\kappa^{q}} \int|v|^{q+1} \phi^{2} .
$$

By (2.3), (2.5) and iterating the computation above with suitable $\phi$, we obtain

$$
\int_{B_{s}}\left[(u-1)_{+}\right]^{q+1} \leq c_{2}\left(s, q, \lambda_{0}, n, p, W, c_{0}, c_{1}\right) \varepsilon^{q+1} .
$$


To derive a contradiction, assume that $u\left(x_{0}\right)-1 \geq \varepsilon^{\eta}$ for some $x_{0} \in B_{s}$. By (3.1), for $y \in B_{\frac{\varepsilon}{2 c_{1}}}\left(x_{0}\right)$,

$$
u(y)-1 \geq u\left(x_{0}\right)-1-\sup |\nabla u| \frac{\varepsilon^{1+\eta}}{2 c_{1}} \geq \frac{\varepsilon^{\eta}}{2} .
$$

Then we have

$$
c_{2} \varepsilon^{q+1} \geq \int_{B_{\frac{\varepsilon^{1+\eta}}{2 c_{1}}}\left(x_{0}\right)}\left[(u-1)_{+}\right]^{q+1} \geq\left(\frac{\varepsilon^{\eta}}{2}\right)^{q+1} \omega_{n}\left(\frac{\varepsilon^{1+\eta}}{2 c_{1}}\right)^{n},
$$

which show by $q=\frac{n p}{n-p}-1$

$$
\varepsilon^{\eta \frac{n p}{n-p}-\frac{n p}{n-p}+n+n \eta} \leq c_{3}\left(s, q, \lambda_{0}, n, p, W, c_{0}, c_{1}\right)
$$

This is a contradiction if $\eta$ and $\varepsilon$ are sufficiently small. $u \geq-1-\varepsilon^{\eta}$ is proved similarly.

The next Lemma 3.3 is the starting point of the ultimate establishment of the monotonicity formula.

Lemma 3.3 For $B_{r}(x) \subset U_{1}$, we have

$$
\begin{gathered}
\frac{d}{d r}\left\{\frac{1}{r^{n-1}} \int_{B_{r}(x)}\left(\frac{\varepsilon|\nabla u|^{2}}{2}+\frac{W(u)}{\varepsilon}\right)\right\}=\frac{1}{r^{n}} \int_{B_{r}(x)}\left(\frac{W(u)}{\varepsilon}-\frac{\varepsilon|\nabla u|^{2}}{2}\right) \\
+\frac{\varepsilon}{r^{n+1}} \int_{\partial B_{r}(x)}((y-x) \cdot \nabla u)^{2}+\frac{\varepsilon}{r^{n}} \int_{B_{r}(x)}(v \cdot \nabla u)((y-x) \cdot \nabla u) .
\end{gathered}
$$

Proof Multiply both sides of (2.1) by $\nabla u \cdot g$, where $g=\left(g^{1}, \ldots, g^{n}\right) \in C_{c}^{1}\left(U_{1} ; \mathbb{R}^{n}\right)$. By integration by parts, we obtain

$$
\int\left(\left(\frac{\varepsilon|\nabla u|^{2}}{2}+\frac{W}{\varepsilon}\right) \operatorname{div} g-\varepsilon \sum_{i, j} u_{y_{i}} u_{y_{j}} g_{y_{j}}^{i}+\varepsilon(v \cdot \nabla u)(\nabla u \cdot g)\right)=0 .
$$

We assume that $x=0$ after a suitable translation and let $g^{j}(y)=y_{j} \rho(|y|)$. Writing $r=|y|$, (3.18) becomes

$$
\begin{aligned}
& \int\left(\left(\frac{\varepsilon|\nabla u|^{2}}{2}+\frac{W}{\varepsilon}\right)\left(r \rho^{\prime}+n \rho\right)-\varepsilon \frac{\rho^{\prime}}{r}(y \cdot \nabla u)^{2}\right. \\
& \left.-\varepsilon|\nabla u|^{2} \rho+\varepsilon(\nabla u \cdot v)(\nabla u \cdot y) \rho\right)=0 .
\end{aligned}
$$

We choose $\rho$ which is a smooth approximation of $\chi_{B_{r}}$, the characteristic function of $B_{r}$, and then we take a limit $\rho \rightarrow \chi_{B_{r}}$. Then we have

$$
\begin{aligned}
- & (n-1) \int_{B_{r}}\left(\frac{\varepsilon|\nabla u|^{2}}{2}+\frac{W}{\varepsilon}\right)+r \int_{\partial B_{r}}\left(\frac{\varepsilon|\nabla u|^{2}}{2}+\frac{W}{\varepsilon}\right) \\
= & \int_{B_{r}}\left(\frac{W}{\varepsilon}-\frac{\varepsilon|\nabla u|^{2}}{2}\right)+\frac{\varepsilon}{r} \int_{\partial B_{r}}(y \cdot \nabla u)^{2}+\varepsilon \int_{B_{r}}(\nabla u \cdot v)(\nabla u \cdot y) .
\end{aligned}
$$

By dividing the above equation by $r^{n}$, the lemma follows. 
We need the following lemma to control the negative contribution of the right-hand side of (3.17).

Lemma 3.4 Given $0<s<1$, there exist constants $0<\beta_{1}<1$ and $0<\varepsilon_{2}<1$ which depend only on $c_{0}, \lambda_{0}, W, n, p$ and such that, if $\varepsilon \leq \varepsilon_{2}$,

$$
\sup _{B_{s}}\left(\frac{\varepsilon}{2}|\nabla u|^{2}-\frac{W(u)}{\varepsilon}\right) \leq \varepsilon^{-\beta_{1}} .
$$

The proof of Lemma 3.4 is deferred to the end of this section. Next, for $B_{r}(x) \subset U_{1}$ and $0<r<\operatorname{dist}\left(x, \partial U_{1}\right)$, define

$$
E(r, x):=\frac{1}{r^{n-1}} \int_{B_{r}(x)}\left(\frac{\varepsilon|\nabla u|^{2}}{2}+\frac{W(u)}{\varepsilon}\right) .
$$

Using Lemmas 3.3 and 3.4, we prove:

Lemma 3.5 Given $0<s<1$, there exist constants $0<\varepsilon_{3}, c_{4}, c_{5}<1$ which depend only on $c_{0}, \lambda_{0}, W, n, p$ and $s$ such that, if $B_{\varepsilon^{\beta_{1}}}(x) \subset U_{s},|u(x)| \leq \alpha$ and $\varepsilon \leq \varepsilon_{3}$, then

$$
E(r, x) \geq c_{4} \text { for all } \varepsilon \leq r \leq c_{5} \varepsilon^{\beta_{1}} .
$$

Proof By integrating (3.17) over $[\varepsilon, r]$, we have

$$
\begin{aligned}
E(r, x)-E(\varepsilon, x) \geq- & \int_{\varepsilon}^{r} \frac{d \tau}{\tau^{n}} \int_{B_{\tau}(x)}\left(\frac{\varepsilon}{2}|\nabla u|^{2}-\frac{W(u)}{\varepsilon}\right)_{+} \\
& +\int_{\varepsilon}^{r} \frac{d \tau}{\tau^{n}} \int_{B_{\tau}(x)} \varepsilon(\nabla u \cdot v)(\nabla u \cdot(y-x)) .
\end{aligned}
$$

By (3.19) and $B_{r}(x) \subset U_{s}$, we have

$$
\int_{\varepsilon}^{r} \frac{d \tau}{\tau^{n}} \int_{B_{\tau}(x)}\left(\frac{\varepsilon}{2}|\nabla u|^{2}-\frac{W(u)}{\varepsilon}\right)_{+} \leq \omega_{n} r \varepsilon^{-\beta_{1}} .
$$

By (3.1) and (2.5), we have

$$
\begin{aligned}
\left|\int_{\varepsilon}^{r} \frac{d \tau}{\tau^{n}} \int_{B_{\tau}(x)} \varepsilon(\nabla u \cdot v)(\nabla u \cdot(y-x))\right| & \leq \int_{0}^{r} \frac{d \tau}{\tau^{n-1}} \int_{B_{\tau}(x)} c_{1}^{2} \varepsilon^{-1}|v| \\
& \leq c\left(\lambda_{0}, n, p, c_{1}\right) r^{3-\frac{n}{p}} \varepsilon^{-1} .
\end{aligned}
$$

Since $|u(x)| \leq \alpha$, using (3.1), we have $|u(y)| \leq \frac{\alpha+1}{2}$ for all $y \in B_{\frac{(1-\alpha) \varepsilon}{2 c_{1}}}(x)$. By choosing a larger $c_{1}$ if necessary, we may assume $\frac{(1-\alpha)}{2 c_{1}} \leq 1$. Define

$$
c_{4}:=\frac{\omega_{n}}{2} \frac{(1-\alpha)^{n}}{\left(2 c_{1}\right)^{n}} \min _{|t| \leq \frac{1+\alpha}{2}} W(t)>0 .
$$

With this choice, we obtain

$$
\begin{aligned}
E(\varepsilon, x) & \geq \frac{1}{\varepsilon^{n-1}} \int_{B_{\frac{(1-\alpha) \varepsilon}{2 c_{1}}}(x)} \frac{W(u)}{\varepsilon} \\
& \geq \omega_{n} \frac{(1-\alpha)^{n}}{\left(2 c_{1}\right)^{n}} \min _{|t| \leq \frac{1+\alpha}{2}} W(t)=2 c_{4} .
\end{aligned}
$$


Since a larger $\beta_{1}$ satisfies (3.19) as well, we may assume $\left(3-\frac{n}{p}\right) \beta_{1}-1>0$ by choosing $\beta_{1}<1$ sufficiently close to 1 . Then we may show that the sum of (3.22) and (3.23) may be bounded from above by $c_{4}$ for sufficiently small $\varepsilon$ and $c_{5}$ if $r \leq c_{5} \varepsilon^{\beta_{1}}$. Then, (3.20) follows from (3.21)-(3.24).

Theorem 3.6 Given $0<s<1$, there exist constants $0<\varepsilon_{4}, \beta_{2}<1$ and $0<c_{6}$ which depend only on $c_{0}, \lambda_{0}, W, n, p$ and s such that, if $B_{r}(x) \subset U_{s}, c_{5} \varepsilon^{\beta_{1}}<r$ and $\varepsilon \leq \varepsilon_{4}$, then

$$
\frac{1}{r^{n}} \int_{B_{r}(x)}\left(\frac{\varepsilon}{2}|\nabla u|^{2}-\frac{W(u)}{\varepsilon}\right)_{+} \leq \frac{c_{6}}{r^{1-\beta_{2}}}(E(r, x)+1) .
$$

Proof The proof is similar to [25, Proposition 3.4] with a minor modification. Define $\beta_{2}:=$ $\frac{1-\beta_{1}}{2 \beta_{1}}$ and $\beta_{3}:=\frac{1+\beta_{1}}{2} . \beta_{2}$ and $\beta_{3}$ are chosen so that

$$
\begin{aligned}
& \beta_{1} \beta_{2}=\beta_{3}-\beta_{1}, \\
& 0<\beta_{2}<1, \quad 0<\beta_{1}<\beta_{3}<1 .
\end{aligned}
$$

We estimate the integral of (3.25) by separating $B_{r}(x)$ into three disjoint sets. Define

$$
\begin{aligned}
& \mathcal{A}:=B_{r}(x) \backslash B_{r-\varepsilon^{\beta_{3}}}(x), \\
& \mathcal{B}:=\left\{y \in B_{r-\varepsilon^{\beta_{3}}}(x): \operatorname{dist}(\{|u| \leq \alpha\}, y)<\varepsilon^{\beta_{3}}\right\}, \\
& \mathcal{C}:=\left\{y \in B_{r-\varepsilon^{\beta_{3}}}(x): \operatorname{dist}(\{|u| \leq \alpha\}, y) \geq \varepsilon^{\beta_{3}}\right\} .
\end{aligned}
$$

Note that $r>c_{5} \varepsilon^{\beta_{1}}>\varepsilon^{\beta_{3}}$ for small $\varepsilon$.

The estimates of the integral over $\mathcal{A}$ and $\mathcal{B}$ are exactly the same as in [25]. Namely, for $\mathcal{A}$, we use $\mathcal{L}^{n}(\mathcal{A}) \leq n \omega_{n} r^{n-1} \varepsilon^{\beta_{3}}$ and (3.19) as well as $r^{-1} \leq\left(c_{5} \varepsilon^{\beta_{1}}\right)^{-1}$. For $\mathcal{B}$, we use (3.20) and prove that $\mathcal{L}^{n}(\mathcal{B}) \leq c(n) \varepsilon^{n \beta_{3}} N$, where $N$ is an integer satisfying $\mathcal{B} \subset \cup_{i=1}^{N} B_{5 \varepsilon^{\beta_{3}}}\left(x_{i}\right)$. Here we only consider the estimate on $\mathcal{C}$ and refer the reader to the proof of [25, Proposition 3.4]. Define

$$
\phi(z):=\min \left\{1, \varepsilon^{-\beta_{3}} \operatorname{dist}(\{y:|y-x| \geq r\} \cup\{|u| \leq \alpha\}, z)\right\} .
$$

$\phi$ is a Lipschitz function and is 0 on $\{y:|y-x|>r\} \cup\{|u|<\alpha\}, 1$ on $\mathcal{C}$ and $|\nabla \phi| \leq \varepsilon^{-\beta_{3}}$. Differentiate (2.1) with respect to $x_{j}$, multiply it by $u_{x_{j}} \phi^{2}$ and sum over $j$. Then we have

$$
\int \sum_{j} \varepsilon u_{x_{j}} \Delta u_{x_{j}} \phi^{2}=\int \frac{W^{\prime \prime}}{\varepsilon}|\nabla u|^{2} \phi^{2}-\varepsilon \sum_{j} \int(v \cdot \nabla u)_{x_{j}} \phi^{2} u_{x_{j}} .
$$

By integration by parts, the Cauchy-Schwarz inequality and (3.1), we obtain

$$
\begin{aligned}
& \int \varepsilon\left|\nabla^{2} u\right|^{2} \phi^{2}+\frac{W^{\prime \prime}}{\varepsilon}|\nabla u|^{2} \phi^{2} \\
& =\int-\sum_{i, j} 2 \varepsilon u_{x_{j}} u_{x_{i} x_{j}} \phi \phi_{x_{i}}-\varepsilon \nabla u \cdot v\left(\Delta u \phi^{2}+2 \phi \nabla u \cdot \nabla \phi\right) \\
& \leq \frac{1}{2} \int \varepsilon\left|\nabla^{2} u\right|^{2} \phi^{2}+c_{7} \int\left(\varepsilon|\nabla u|^{2}|\nabla \phi|^{2}+|v|^{2} \phi^{2} \varepsilon^{-1}\right),
\end{aligned}
$$

where $c_{7}$ depends on $c_{1}$. Since $|u| \geq \alpha$ on the support of $\phi$, we have $W^{\prime \prime} \geq \kappa$. Thus

$$
\int \frac{\varepsilon}{2}\left|\nabla^{2} u\right|^{2} \phi^{2}+\frac{\kappa}{\varepsilon}|\nabla u|^{2} \phi^{2} \leq c_{7} \int\left(\varepsilon|\nabla u|^{2}|\nabla \phi|^{2}+|v|^{2} \phi^{2} \varepsilon^{-1}\right) .
$$


By $|\nabla \phi| \leq \varepsilon^{-\beta_{3}}$ and (2.5), we have

$$
\begin{aligned}
\int \frac{\kappa}{\varepsilon}|\nabla u|^{2} \phi^{2} & \leq c_{7}\left(\varepsilon^{-2 \beta_{3}} \int_{B_{r}} \varepsilon|\nabla u|^{2}+\varepsilon^{-1}\|v\|_{L^{\frac{n p}{n-p}}}^{2}\left(\omega_{n} r^{n}\right)^{\frac{n p-2(n-p)}{n p}}\right) \\
& \leq c_{8}\left(\varepsilon^{-2 \beta_{3}} \int_{B_{r}} \varepsilon|\nabla u|^{2}+\varepsilon^{-1} r^{n-\frac{2(n-p)}{p}}\right),
\end{aligned}
$$

where $c_{8}=c_{7}+c(n, p) \lambda_{0}^{2}$. Since $\phi=1$ on $\mathcal{C}$, multiplying (3.31) by $\frac{\varepsilon^{2}}{\kappa r^{n}}$,

$$
\frac{1}{r^{n}} \int_{\mathcal{C}} \frac{\varepsilon}{2}|\nabla u|^{2} \leq \frac{c_{8}}{\kappa}\left(\frac{\varepsilon^{2-2 \beta_{3}}}{r} E(r, x)+\varepsilon r^{2-\frac{2 n}{p}}\right) .
$$

By the definitions of $\beta_{1}, \beta_{2}, \beta_{3}$ and $r \geq c_{5} \varepsilon^{\beta_{1}}$, we have

$$
\frac{\varepsilon^{2-2 \beta_{3}}}{r} \leq \frac{\varepsilon^{\beta_{1} \beta_{2}}}{r^{1-\beta_{2}} c_{5}^{\beta_{2}}},
$$

and using $\varepsilon \leq r$,

$$
\varepsilon r^{2-\frac{2 n}{p}} \leq \frac{1}{r^{\frac{2 n}{p}-3}},
$$

where $\frac{2 n}{p}-3<1$. Hence, we obtain

$$
\frac{1}{r^{n}} \int_{\mathcal{C}} \frac{\varepsilon}{2}|\nabla u|^{2} \leq \frac{c_{8}}{\kappa}\left(\frac{\varepsilon^{\beta_{1} \beta_{2}}}{r^{1-\beta_{2}} c_{5}^{\beta_{2}}} E(r, x)+\frac{1}{r^{\frac{2 n}{p}-3}}\right) .
$$

By re-defining $\beta_{2}=\min \left\{\beta_{2}, 4-\frac{2 n}{p}\right\}$ and the estimates of integrals over $\mathcal{A}, \mathcal{B}$ and $\mathcal{C}$, we proved (3.25).

To proceed, we need the following theorem (see [30, Theorem 5.12.4]).

Theorem 3.7 Let $\mu$ be a positive Radon measure on $\mathbb{R}^{n}$ satisfying

$$
K(\mu):=\sup _{B_{r}(x) \subset \mathbb{R}^{n}} \frac{1}{r^{n-1}} \mu\left(B_{r}(x)\right)<\infty .
$$

Then there exists a constant $c(n)$ such that

$$
\left|\int_{\mathbb{R}^{n}} \phi d \mu\right| \leq c(n) K(\mu) \int_{\mathbb{R}^{n}}|\nabla \phi| d \mathcal{L}^{n}
$$

for all $\phi \in C_{c}^{1}\left(\mathbb{R}^{n}\right)$.

Theorem 3.8 There exists a constant $0<c_{9}$ which depends only on $c_{0}, \lambda_{0}, W, E_{0}, n$ and $p$ such that, if $0<\varepsilon<1 / 2$ and $U_{2 r}(x) \subset U_{1-\varepsilon}$, then

$$
\operatorname{dist}\left(x, \partial U_{1-\varepsilon}\right)^{n-1} E(r, x) \leq c_{9} .
$$

Proof Define

$$
E_{1}:=\sup _{U_{2 r}(x) \subset U_{1-\varepsilon}} \operatorname{dist}\left(x, \partial U_{1-\varepsilon}\right)^{n-1} E(r, x) .
$$


By Lemma 3.1, we have $\sup _{x \in U_{1-\varepsilon}} \varepsilon|\nabla u(x)| \leq c_{1}$. Thus for any $U_{2 r}(x) \subset U_{1-\varepsilon}$, we have

$$
E(r, x) \leq \omega_{n} r\left(\frac{c_{1}^{2}}{2 \varepsilon}+\sup _{|t| \leq c_{0}} \frac{W(t)}{\varepsilon}\right) \leq \frac{c\left(n, c_{1}, W\right)}{\varepsilon}
$$

and we have $E_{1}<\infty$ for each $\varepsilon$. In the following, we give an estimate of $E_{1}$ depending only on $c_{0}, \lambda_{0}, n, p, W$ and $E_{0}$. Let $U_{2 r_{0}}\left(x_{0}\right) \subset U_{1-\varepsilon}$ be fixed such that

$$
\operatorname{dist}\left(x_{0}, \partial U_{1-\varepsilon}\right)^{n-1} E\left(r_{0}, x_{0}\right)>\frac{3}{4} E_{1} \text {. }
$$

For simplicity, define

$$
l:=\operatorname{dist}\left(x_{0}, \partial U_{1-\varepsilon}\right)=1-\varepsilon-\left|x_{0}\right|
$$

and change variables by $\tilde{x}=\left(x-x_{0}\right) / l, \tilde{r}=r / l, \tilde{\varepsilon}=\varepsilon / l, \tilde{u}(\tilde{x})=u(x)$ and $\tilde{v}(\tilde{x})=l v(x)$. Note that $U_{l+\varepsilon}\left(x_{0}\right) \subset U_{1}$. In particular, we write

$$
\tilde{r}_{0}:=r_{0} / l \leq 1 / 2 \text {. }
$$

By (2.1), (2.4) and (2.5), we have

$$
\begin{aligned}
& -\tilde{\varepsilon} \Delta \tilde{u}+\frac{W^{\prime}(\tilde{u})}{\tilde{\varepsilon}}=\tilde{\varepsilon} \tilde{v} \cdot \nabla \tilde{u} \text { for } \tilde{x} \in U_{1+\tilde{\varepsilon}}, \\
& \int_{U_{1+\tilde{\varepsilon}}}\left(\frac{\tilde{\varepsilon}|\nabla \tilde{u}|^{2}}{2}+\frac{W(\tilde{u})}{\tilde{\varepsilon}}\right) \leq l^{1-n} E_{0}, \\
& \|\tilde{v}\|_{L^{\frac{n p}{n-p}}\left(U_{1+\tilde{\varepsilon})}\right.}+\|\nabla \tilde{v}\|_{L^{p}\left(U_{1+\tilde{\varepsilon}}\right)} \leq l^{2-\frac{n}{p}} \lambda_{0} .
\end{aligned}
$$

Define for $B_{\tilde{r}}(\tilde{x}) \subset U_{1+\tilde{\varepsilon}}$

$$
\tilde{E}(\tilde{r}, \tilde{x}):=\frac{1}{\tilde{r}^{n-1}} \int_{B_{\tilde{r}}(\tilde{x})}\left(\frac{\tilde{\varepsilon}|\nabla \tilde{u}|^{2}}{2}+\frac{W(\tilde{u})}{\tilde{\varepsilon}}\right) .
$$

Under the above change of variables, note that we have $E(r, x)=\tilde{E}(\tilde{r}, \tilde{x})$. Next, for any $x \in B_{3 l / 4}\left(x_{0}\right)$, we have dist $\left(x, \partial U_{1-\varepsilon}\right) \geq l / 4$. Hence for any $x \in B_{3 l / 4}\left(x_{0}\right)$ and $r<l / 8 \leq$ dist $\left(x, \partial U_{1-\varepsilon}\right) / 2$, by the definition of $E_{1}$, we have

$$
\operatorname{dist}\left(x, \partial U_{1-\varepsilon}\right)^{n-1} E(r, x) \leq E_{1} .
$$

This shows (again using $\left.\operatorname{dist}\left(x, \partial U_{1-\varepsilon}\right) \geq l / 4\right)$

$$
\sup _{\tilde{x} \in B_{\frac{3}{4}}, 0<\tilde{r}<\frac{1}{8}} \tilde{E}(\tilde{r}, \tilde{x}) \leq 4^{n-1} l^{1-n} E_{1} .
$$

We next let $c_{1}, c_{4}, c_{5}, c_{6}, \varepsilon_{1}, \varepsilon_{2}, \varepsilon_{3}, \varepsilon_{4}, \beta_{1}, \beta_{2}$ be constants obtained in Lemma 3.1-3.5 and Theorem 3.6 corresponding to the same $c_{0}, \lambda_{0}, n, p, W$ and $s=3 / 4$. Then note that the estimates up to Theorem 3.6 hold for $\tilde{u}$ and $\tilde{v}$ for $U_{3 / 4}$ and with respect to the new variables $(\tilde{x}, \tilde{r}, \tilde{\varepsilon}$ etc. $)$ if

$$
\tilde{\varepsilon} \leq \hat{\varepsilon}:=\min \left\{\varepsilon_{1}, \varepsilon_{2}, \varepsilon_{3}, \varepsilon_{4}, 1 / 2\right\}
$$

due to (3.38) and (3.40). It is important to note that $\hat{\varepsilon}$ depends only on $c_{0}, \lambda_{0}, n, p$ and $W$. Note that (3.40) yields an upper bound for $\|\tilde{v}\|_{L^{\frac{n p}{n-p}}\left(U_{1+\tilde{\varepsilon})}\right.}+\|\nabla \tilde{v}\|_{L^{p}\left(U_{1+\tilde{\varepsilon})}\right.}$ independent of $l$, 
as $l<1$ and $2-\frac{n}{p}>0$. A priori, we do not know if (3.43) holds or not and we prove the desired estimate for $E_{1}$ by exhausting all the possibilities.

First consider the case $\tilde{\varepsilon} \geq \hat{\varepsilon}$. We use (3.3) and (3.1), respectively, for $\tilde{\varepsilon}>1 / 2$ and $1 / 2 \geq \tilde{\varepsilon} \geq \hat{\varepsilon}$. Suppose that $\tilde{\varepsilon}>1 / 2$. By (3.37) and (3.3), we have

$$
\frac{3}{4 l^{n-1}} E_{1}<\tilde{E}\left(\tilde{r}_{0}, 0\right) \leq \omega_{n} \tilde{r}_{0}\left(\tilde{\varepsilon} c_{1}^{2}+2 \sup _{|x| \leq c_{0}} W(x)\right)
$$

and since $l \tilde{\varepsilon}=\varepsilon \leq 1, l<1$ and $\tilde{r}_{0} \leq 1 / 2$, we obtain

$$
E_{1}<\frac{4}{3} \omega_{n}\left(c_{1}^{2}+2 \sup _{|x| \leq c_{0}} W(x)\right) .
$$

If $1 / 2 \geq \tilde{\varepsilon} \geq \hat{\varepsilon}$, again by (3.37), (3.1) and $\tilde{r}_{0} \leq 1 / 2$, we have

$$
\begin{aligned}
\frac{3}{4 l^{n-1}} E_{1} & <\tilde{E}\left(\tilde{r}_{0}, 0\right) \leq \omega_{n}\left(c_{1}^{2}+\sup _{|x| \leq c_{0}} W(x)\right) \frac{\tilde{r}_{0}}{\tilde{\varepsilon}} \\
& \leq \omega_{n}\left(c_{1}^{2}+\sup _{|x| \leq c_{0}} W(x)\right) \frac{1}{2 \hat{\varepsilon}}
\end{aligned}
$$

and we obtain

$$
E_{1}<\omega_{n}\left(c_{1}^{2}+\sup _{|x| \leq c_{0}} W(x)\right) \frac{2}{3 \hat{\varepsilon}} .
$$

Thus by (3.44) and (3.45), if $\tilde{\varepsilon} \geq \hat{\varepsilon}, E_{1}$ is bounded by a constant which depends only on $c_{0}, \lambda_{0}, n, p$ and $W$.

For the rest of the proof, consider the case $\tilde{\varepsilon}<\hat{\varepsilon}$ and consider the following four cases (a)-(d) depending on how large $\tilde{r}_{0}=r_{0} / l$ is relative to $\tilde{\varepsilon}$ and $\tilde{r}_{1}$, where $\tilde{r}_{1}$ will be determined shortly depending only on $c_{0}, \lambda_{0}, n, p, W$ and $E_{0}$ :

$$
\text { (a) } \tilde{r}_{1}<\tilde{r}_{0} \leq \frac{1}{2}, \quad(b) c_{5} \tilde{\varepsilon}^{\beta_{1}}<\tilde{r}_{0} \leq \tilde{r}_{1}, \quad(c) \tilde{\varepsilon}<\tilde{r}_{0} \leq c_{5} \tilde{\varepsilon}^{\beta_{1}}, \quad(d) 0<\tilde{r}_{0} \leq \tilde{\varepsilon}
$$

To control the term involving $v$ in (3.17), define a Radon measure

$$
\mu(A):=\int_{A \cap B_{\frac{3}{4}}}\left(\frac{\tilde{\varepsilon}|\nabla \tilde{u}|^{2}}{2}+\frac{W(\tilde{u})}{\tilde{\varepsilon}}\right) .
$$

By Theorem 3.7 and (3.42) (note that (3.42) has the restriction $\tilde{r}<1 / 8$ but this can be dropped easily by replacing $4^{n-1}$ by a larger constant depending only on $n$ ), we have

$$
\left|\int_{B_{\frac{3}{4}}} \phi d \mu\right| \leq c(n) l^{1-n} E_{1} \int_{\mathbb{R}^{n}}|\nabla \phi| d \mathcal{L}^{n}
$$

for all $\phi \in C_{c}^{1}\left(\mathbb{R}^{n}\right)$. By (3.17) and (3.25), if $c_{5} \tilde{\varepsilon}^{\beta_{1}}<\tilde{r} \leq \frac{1}{2}$, we have

$$
\begin{aligned}
\frac{d}{d \tilde{r}} \tilde{E}(\tilde{r}, 0) \geq & -\frac{c_{6}}{\tilde{r}^{1-\beta_{2}}}(\tilde{E}(\tilde{r}, 0)+1)-\frac{1}{\tilde{r}^{n-1}} \int_{B_{\tilde{r}}} \tilde{\varepsilon}|\tilde{v}||\nabla \tilde{u}|^{2} \\
& +\frac{1}{\tilde{r}^{n}} \int_{B_{\tilde{r}}}\left(\frac{W(\tilde{u})}{\tilde{\varepsilon}}-\frac{\tilde{\varepsilon}}{2}|\nabla \tilde{u}|^{2}\right)_{+} .
\end{aligned}
$$


Let $\phi \in C_{c}^{1}\left(B_{\frac{3 \tilde{r}}{2}}\right)$ be such that $0 \leq \phi \leq 1, \phi(y)=1$ in $B_{\tilde{r}}$ and $|\nabla \phi| \leq \frac{4}{\tilde{r}}$. We use (3.46) with (3.40) by smoothly approximating $|\tilde{v}|$ as

$$
\begin{gathered}
\int_{B_{\tilde{r}}} \tilde{\varepsilon}|\tilde{v}||\nabla \tilde{u}|^{2} \leq \int_{B_{\frac{3 \tilde{r}}{2}}} \tilde{\varepsilon} \phi|\tilde{v}||\nabla \tilde{u}|^{2} \leq c(n) l^{1-n} E_{1} \int_{B_{\frac{3 \tilde{r}}{2}}}|\nabla(\phi|\tilde{v}|)| \\
\leq c(n) l^{1-n} E_{1} \int_{B_{\frac{3 \tilde{r}}{2}}} \frac{4}{\tilde{r}}|\tilde{v}|+|\nabla \tilde{v}| \leq c(n) l^{3-n-\frac{n}{p}} \lambda_{0} \tilde{r}^{n-\frac{n}{p}} E_{1} .
\end{gathered}
$$

Hence, for $c_{5} \tilde{\varepsilon}^{\beta_{1}}<\tilde{r} \leq \frac{1}{2}$, (3.47) with (3.48) and (3.42) give

$$
\begin{aligned}
\frac{d}{d \tilde{r}} \tilde{E}(\tilde{r}, 0) \geq & -c_{6} \tilde{r}^{\beta_{2}-1}\left(c(n) l^{1-n} E_{1}+1\right)-c(n) l^{3-n-\frac{n}{p}} \lambda_{0} \tilde{r}^{1-\frac{n}{p}} E_{1} \\
& +\frac{1}{\tilde{r}^{n}} \int_{B_{\tilde{r}}}\left(\frac{W(\tilde{u})}{\tilde{\varepsilon}}-\frac{\tilde{\varepsilon}}{2}|\nabla \tilde{u}|^{2}\right)_{+} .
\end{aligned}
$$

By integrating (3.49) over $\tilde{r} \in\left(\tilde{s}_{1}, \tilde{s}_{2}\right)$ with $c_{5} \tilde{\varepsilon}^{\beta_{1}}<\tilde{s}_{1}<\tilde{s}_{2} \leq \frac{1}{2}$, we obtain

$$
\begin{aligned}
\tilde{E}\left(\tilde{s}_{2}, 0\right)-\tilde{E}\left(\tilde{s}_{1}, 0\right) \geq & -c_{10}\left(\tilde{s}_{2}^{\beta_{2}}+l^{2-\frac{n}{p}} \tilde{s}_{2}^{2-\frac{n}{p}}\right) l^{1-n} E_{1}-c_{10} \tilde{s}_{2}^{\beta_{2}} \\
& +\int_{\tilde{s}_{1}}^{\tilde{s}_{2}} \frac{d \tilde{r}}{\tilde{r}^{n}} \int_{B_{\tilde{r}}}\left(\frac{W(\tilde{u})}{\tilde{\varepsilon}}-\frac{\tilde{\varepsilon}}{2}|\nabla \tilde{u}|^{2}\right)_{+},
\end{aligned}
$$

where $c_{10}$ depends only on $c_{0}, \lambda_{0}, n, p$ and $W$. At this point, we choose $\tilde{r}_{1}<1 / 2$ depending only on $c_{0}, \lambda_{0}, n, p$ and $W$ so that

$$
c_{10}\left(\tilde{r}_{1}^{\beta_{2}}+\tilde{r}_{1}^{2-\frac{n}{p}}\right)<\frac{1}{4} .
$$

This in particular implies from (3.50) that if $c_{5} \tilde{\varepsilon}^{\beta_{1}}<\tilde{s}_{1}<\tilde{s}_{2} \leq \tilde{r}_{1}$, then

$$
\tilde{E}\left(\tilde{s}_{2}, 0\right)-\tilde{E}\left(\tilde{s}_{1}, 0\right) \geq-c_{10}-\frac{1}{4} l^{1-n} E_{1} .
$$

With this $\tilde{r}_{1}$ being fixed, we proceed to check that $E_{1}$ is bounded in terms of $c_{0}, \lambda_{0}, n, p, W, E_{0}$ in each case (a)-(d).

Case (a): By (3.37), (3.39) and $\tilde{r}_{1}<\tilde{r}_{0}$, we have

$$
\frac{3}{4} l^{1-n} E_{1} \leq \tilde{E}\left(\tilde{r}_{0}, 0\right) \leq \tilde{r}_{0}^{1-n} l^{1-n} E_{0} \leq \tilde{r}_{1}^{1-n} l^{1-n} E_{0} .
$$

Hence

$$
E_{1} \leq \frac{4}{3} \tilde{r}_{1}^{1-n} E_{0}
$$

and $E_{1}$ is bounded by a constant depending only on $c_{0}, \lambda_{0}, n, p, W$ and $E_{0}$.

Case (b): Since $c_{5} \tilde{\varepsilon}^{\beta_{1}}<\tilde{r}_{0} \leq \tilde{r}_{1}$, we may use (3.51) with $\tilde{s}_{2}=\tilde{r}_{1}$ and $\tilde{s}_{1}=\tilde{r}_{0}$. Then we obtain

$$
\tilde{E}\left(\tilde{r}_{1}, 0\right)-\tilde{E}\left(\tilde{r}_{0}, 0\right) \geq-c_{10}-\frac{1}{4} l^{1-n} E_{1} .
$$

Then, by (3.37) and (3.39), we obtain

$$
E_{1} \leq 4\left(\tilde{E}\left(\tilde{r}_{1}, 0\right)+c_{10}\right) l^{n-1} \leq 4\left(\tilde{r}_{1}^{1-n} E_{0}+c_{10}\right)
$$


which depends only on $c_{0}, \lambda_{0}, n, p, W$ and $E_{0}$.

Case (c): By the same estimate used in the proof of Lemma 3.5, we have

$$
\tilde{E}\left(c_{5} \tilde{\varepsilon}^{\beta_{1}}, 0\right)-\tilde{E}\left(\tilde{r}_{0}, 0\right) \geq-c_{4} .
$$

We use (3.51) with $\tilde{s}_{1}=c_{5} \tilde{\varepsilon}^{\beta_{1}}$ and $\tilde{s}_{2}=\tilde{r}_{1}$ to obtain

$$
\tilde{E}\left(\tilde{r}_{1}, 0\right)-\tilde{E}\left(c_{5} \tilde{\varepsilon}^{\beta_{1}}, 0\right) \geq-c_{10}-\frac{1}{4} l^{1-n} E_{1},
$$

and (3.52) and (3.53) combined with (3.37) give

$$
E_{1} \leq 4 l^{n-1}\left(\tilde{E}\left(\tilde{r}_{1}, 0\right)+c_{4}+c_{10}\right) \leq 4 \tilde{r}_{1}^{1-n} E_{0}+4\left(c_{4}+c_{10}\right),
$$

which depends only on $c_{0}, \lambda_{0}, n, p, W$ and $E_{0}$.

Case (d): Since $\tilde{r}_{0} \leq \tilde{\varepsilon}$, we use (3.1) to obtain

$$
\tilde{E}\left(\tilde{r}_{0}, 0\right) \leq \omega_{n}\left(c_{1}^{2}+\sup _{|x| \leq c_{0}} W(x)\right) \frac{\tilde{r}_{0}}{\tilde{\varepsilon}} \leq \omega_{n}\left(c_{1}^{2}+\sup _{|x| \leq c_{0}} W(x)\right) .
$$

Then (3.54) and (3.37) gives

$$
E_{1}<\frac{4}{3} \omega_{n}\left(c_{1}^{2}+\sup _{|x| \leq c_{0}} W(x)\right) l^{n-1} \leq \omega_{n}\left(c_{1}^{2}+\sup _{|x| \leq c_{0}} W(x)\right) .
$$

This completes the estimate for $E_{1}$.

Once we obtain the upper density estimate, we may obtain the following monotonicity formula.

Theorem 3.9 Given $0<s<1$, there exist constants $0<c_{11}$ and $0<\varepsilon_{5}<1$ depending only on $c_{0}, \lambda_{0}, n, p, W, E_{0}$ and $s$, such that, if $c_{5} \varepsilon^{\beta_{1}} \leq s_{1}<s_{2}, B_{s_{2}}(x) \subset U_{s}$ and $\varepsilon<\varepsilon_{5}$, then

$$
\begin{aligned}
E\left(s_{2}, x\right)-E\left(s_{1}, x\right) \geq & -c_{11}\left(s_{2}^{2-\frac{n}{p}}+s_{2}^{\beta_{2}}\right) \\
& +\int_{s_{1}}^{s_{2}} \frac{d \tau}{\tau^{n}} \int_{B_{\tau}(x)}\left(\frac{W(u)}{\varepsilon}-\frac{\varepsilon}{2}|\nabla u|^{2}\right)_{+} .
\end{aligned}
$$

Proof Let $\varepsilon_{5}=\min \left\{\varepsilon_{1}, \varepsilon_{2}, \varepsilon_{3}, \varepsilon_{4},(1-s) / 2\right\}$ corresponding to the given $s$ and suppose that $\varepsilon<\varepsilon_{5}$. For any $x \in U_{s}$ and $0<r<(1-s) / 2$, by Theorem 3.8, $E(r, x) \leq c_{9}(1-s-\varepsilon)^{1-n}$, where the right-hand side is bounded by a constant depending only on $c_{0}, \lambda_{0}, n, p, W, E_{0}$ and $s$. For $B_{s_{2}}(x) \subset U_{s}$, we have (3.25) and (3.17). Arguing as (3.46)-(3.50) without change of variables (so $l=1$ ) and with $\mu$ restricted to $B_{s}$ in place of $B_{3 / 4}$, we obtain (3.55).

Theorem 3.10 Given $0<s<1$, there exist constants $0<c_{12}$ depending only on $c_{0}, \lambda_{0}, n, p, W, E_{0}$ and s such that, if $\varepsilon<\varepsilon_{5},|u(x)| \leq \alpha, \varepsilon \leq r$ and $B_{r}(x) \subset U_{s}$, then

$$
E(r, x) \geq c_{12} .
$$


Proof By Lemma 3.5, we may assume $c_{5} \tilde{\varepsilon}^{\beta_{1}} \leq r$ and $E\left(c_{5} \varepsilon^{\beta_{1}}, x\right) \geq c_{4}$. In (3.55), let $s_{1}=c_{5} \varepsilon^{\beta_{1}}$ and $s_{2}=r$. Fix $r_{1}>0$ depending only on $c_{0}, \lambda_{0}, n, p, \bar{W}, E_{0}$ and $s$ so that $c_{11}\left(r_{1}^{2-\frac{n}{p}}+r_{1}^{\beta_{2}}\right) \leq c_{4} / 2$. Then for $c_{5} \varepsilon^{\beta_{1}} \leq r \leq r_{1}$, (3.55) shows that $E(r, x) \geq c_{4} / 2$. For $1>r>r_{1}, E(r, x) \geq r_{1}^{n-1} E\left(r_{1}, x\right) \geq r_{1}^{n-1} c_{4} / 2$. Thus, setting $c_{12}=r_{1}^{n-1} c_{4} / 2$, we have (3.56).

For the rest of the present section, we finish the proof of Lemma 3.4. We use the following result proved in [25, Lemma 3.9].

Lemma 3.11 Given $0<\eta, \beta_{4}<1, \eta \leq \beta_{4}, 0<c_{13}$, there exist $\varepsilon_{6}>0, c_{14}>0$ depending only on $\eta, \beta_{4}, c_{13}, n$ and $W$ with the following properties: Suppose $f \in C^{3}\left(U_{\varepsilon^{-\beta_{4}}}\right), g \in$ $C^{1}\left(U_{\varepsilon^{-\beta_{4}}}\right)$ and $0<\varepsilon \leq \varepsilon_{6}$ satisfy

$$
-\Delta f+W^{\prime}(f)=g
$$

on $U_{\varepsilon^{-\beta_{4}}}$ and

$$
\sup _{U_{\varepsilon}-\beta_{4}}|f| \leq 1+\varepsilon^{\eta}, \quad \sup _{U_{\varepsilon}-\beta_{4}}\left(\frac{1}{2}|\nabla f|^{2}-W(f)\right) \leq c_{13} .
$$

Then

$$
\sup _{\frac{B_{\frac{\varepsilon}{2}-\beta_{4}}}{2}}\left(\frac{1}{2}|\nabla f|^{2}-W(f)\right) \leq c_{13}\left(\varepsilon^{-\beta_{4}}\|g\|_{W^{1, n}\left(B_{\varepsilon}-\beta_{4}\right)}+\varepsilon^{\eta}\right) .
$$

We remark that the assumptions on $W$ are essentially used for the proof of Lemma 3.11.

Proof of Lemma 3.4 As in the proof of Lemma 3.1, define $\tilde{u}(x):=u(\varepsilon x), \tilde{v}(x):=\varepsilon v(\varepsilon x)$, and subsequently drop $\sim$ for simplicity. We have

$$
-\Delta u+W^{\prime}(u)=\nabla u \cdot v
$$

on $U_{\varepsilon^{-1}}$. With respect to the new variables, we need to prove

$$
\sup _{U_{s \varepsilon^{-1}}}\left(\frac{1}{2}|\nabla u|^{2}-W(u)\right) \leq \varepsilon^{1-\beta_{1}}
$$

for some $0<\beta_{1}<1$ for all sufficiently small $\varepsilon$. Let $\phi_{\lambda}$ be the standard mollifier, namely, define

$$
\phi(x):= \begin{cases}C \exp \left(\frac{1}{|x|^{2}-1}\right) & \text { for }|x|<1 \\ 0 & \text { for }|x| \geq 1\end{cases}
$$

where the constant $C>0$ is selected so that $\int_{\mathbb{R}^{n}} \phi=1$, and define $\phi_{\lambda}(x):=\frac{1}{\lambda^{n}} \phi\left(\frac{x}{\lambda}\right)$. For $0<\beta_{5}<1$ to be chosen depending only on $n$ and $p$ later, define for $x \in U_{\varepsilon^{-1}-1}$

$$
f(x):=\left(u * \phi_{\varepsilon} \beta_{5}\right)(x)=\int u(x-y) \phi_{\varepsilon} \beta_{5}(y) d y .
$$

By (3.1) and (3.2), we have

$$
\begin{gathered}
\sup _{U_{\varepsilon^{-1}-1}}|f-u| \leq 2 c_{1} \varepsilon^{\beta_{5}}, \\
\sup _{U_{\varepsilon^{-1}-1}}|\nabla f-\nabla u| \leq 2 c_{1} \varepsilon^{\beta_{5}}\left(2-\frac{n}{p}\right) .
\end{gathered}
$$


We next define $g$ to be

$$
g:=(\nabla u \cdot v) * \phi_{\varepsilon} \beta_{5}+\left(W^{\prime}(f)-W^{\prime}(u) * \phi_{\varepsilon} \beta_{5}\right),
$$

so that we have

$$
-\Delta f+W^{\prime}(f)=g .
$$

To use Lemma 3.11, we next estimate the $W^{1, n}$ norm of $g$ on $U_{\varepsilon^{-\beta_{4}}}(x)$ with $x \in U_{s \varepsilon^{-1}}$, where $0<\beta_{4}<1$ will be chosen depending only on $n$ and $p$. In the following, let us write $U_{\varepsilon^{-\beta_{4}}}(x)$ as $U_{\varepsilon^{-\beta_{4}}}$ and $U_{\frac{\varepsilon-\beta_{4}}{2}}(x)$ as $U_{\frac{\varepsilon}{2}-\beta_{4}}$ for simplicity. For sufficiently small $\varepsilon$ depending on $s$ and $\beta_{4}$ so that $U_{2 \varepsilon^{-\beta_{4}}}(x) \subset U_{\varepsilon^{-1}-1}$ (and so that we may use (3.1)), the first term of (3.62) can be estimated as

$$
\left.\left\|(\nabla u \cdot v) * \phi_{\varepsilon^{\beta}}\right\|_{W^{1, n}\left(U_{\varepsilon^{-}-\beta_{4}}\right)} \leq c_{15}\left(1+\varepsilon^{-\beta_{5}}\right)\|v\|_{L^{n}\left(U_{2 \varepsilon}-\beta_{4}\right.}\right)
$$

where $c_{15}$ depends only on $\phi, n$ and $c_{1}$. By (3.5), we obtain

$$
\begin{aligned}
\|v\|_{L^{n}\left(U_{2 \varepsilon}-\beta_{4}\right)} & \leq\|v\|_{L^{\frac{n p}{n-p}}\left(U_{2 \varepsilon}-\beta_{4}\right)}\left\{\omega_{n}\left(2 \varepsilon^{-\beta_{4}}\right)^{n}\right\}^{\frac{2 p-n}{n p}} \\
& \leq \lambda_{0} \varepsilon^{\left(2-\frac{n}{p}\right)\left(1-\beta_{4}\right)}\left(2^{n} \omega_{n}\right)^{\frac{2 p-n}{n p}} .
\end{aligned}
$$

Thus (3.64) and (3.65) show

$$
\left\|(\nabla u \cdot v) * \phi_{\varepsilon} \beta_{5}\right\|_{W^{1, n}\left(U_{\varepsilon}-\beta_{4}\right)} \leq c_{16} \varepsilon^{\left(2-\frac{n}{p}\right)\left(1-\beta_{4}\right)-\beta_{5}}
$$

where $c_{16}$ depends only on $\phi, n, p, \lambda_{0}$ and $c_{1}$. We next consider the second term of (3.62). By (3.60), (3.61) and

$$
W^{\prime}(f)-W^{\prime}(u) * \phi_{\varepsilon}^{\beta_{5}}=\left(W^{\prime}(f)-W^{\prime}(u)\right)+\left(W^{\prime}(u)-W^{\prime}(u) * \phi_{\varepsilon} \beta_{5}\right),
$$

we compute

$$
\begin{aligned}
& \sup \left|W^{\prime}(f)-W^{\prime}(u)\right| \leq \sup \left|W^{\prime \prime}\right| \sup |u-f| \leq c_{17} \varepsilon^{\beta_{5}}, \\
& \sup \left|\nabla\left(W^{\prime}(f)-W^{\prime}(u)\right)\right| \leq \sup \left|W^{\prime \prime}\right| \sup |\nabla f-\nabla u| \\
& \quad+\sup |\nabla u| \sup \left|W^{\prime \prime \prime}\right| \sup |u-f| \\
& \quad \leq c_{17} \varepsilon^{\beta_{5}}\left(2-\frac{n}{p}\right), \\
& \sup \left|W^{\prime}(u)-W^{\prime}(u) * \phi_{\varepsilon} \beta_{5}\right| \leq c_{17} \varepsilon^{\beta_{5}}, \\
& \sup \left|\nabla\left(W^{\prime}(u)-W^{\prime}(u) * \phi_{\varepsilon} \beta_{5}\right)\right| \leq c_{17} \varepsilon^{\beta_{5}}\left(2-\frac{n}{p}\right) .
\end{aligned}
$$

Here $c_{17}$ depends only on $\phi, n, \lambda_{0}, c_{1}$ and $W$. Hence, (3.67)-(3.70) show

$$
\left\|W^{\prime}(f)-W^{\prime}(u) * \phi_{\varepsilon} \beta_{5}\right\|_{W^{1, n}\left(U_{\varepsilon}-\beta_{4}\right) \leq 4 c_{17} \varepsilon^{\beta_{5}}\left(2-\frac{n}{p}\right)-\beta_{4}} .
$$

By (3.62), (3.66) and (3.71), we have

$$
\|g\|_{W^{1, n}\left(U_{\varepsilon}-\beta_{4}\right)} \leq c_{16} \varepsilon^{\left(2-\frac{n}{p}\right)\left(1-\beta_{4}\right)-\beta_{5}}+4 c_{17} \varepsilon^{\beta_{5}}\left(2-\frac{n}{p}\right)-\beta_{4} .
$$

We use Lemma 3.11 to $f$ and $g$. Due to Lemma 3.2, we have $\sup |f| \leq \sup |u| \leq 1+\varepsilon^{\eta}$ on $U_{\varepsilon^{-\beta_{4}}}$ and we may choose smaller $\eta$ if necessary. Because of (3.1), we have $c_{13} \geq$ $\sup _{U_{\varepsilon}-\beta_{4}}\left(\frac{1}{2}|\nabla f|^{2}-W(f)\right)$ for a constant depending only on $c_{1}$ and $W$ (here again we 
restrict $\varepsilon$ small so that $\left.U_{\varepsilon^{-\beta_{4}}}(x) \subset U_{\varepsilon^{-1}-1} \cap U_{\varepsilon^{-1}(1+s) / 2}\right)$. Then we have all the assumptions for Lemma 3.11 and obtain

$$
\begin{aligned}
\sup _{\frac{U_{\frac{\varepsilon}{2}-\beta_{4}}}{2}} & \left(\frac{1}{2}|\nabla f|^{2}-W(f)\right) \\
& \leq c_{14}\left(\varepsilon^{\left(2-\frac{n}{p}\right)\left(1-\beta_{4}\right)-\beta_{4}-\beta_{5}}+\varepsilon^{\beta_{5}}\left(2-\frac{n}{p}\right)-2 \beta_{4}+\varepsilon^{\eta}\right) .
\end{aligned}
$$

At this point, we fix sufficiently small $0<\beta_{4}, \beta_{5}<1$ depending only on $n$ and $p$ such that

$$
\left(2-\frac{n}{p}\right)\left(1-\beta_{4}\right)-\beta_{4}-\beta_{5}>0, \quad \beta_{5}\left(2-\frac{n}{p}\right)-2 \beta_{4}>0 .
$$

This shows that we may choose a $0<\beta_{1}<1$ such that

$$
\sup _{\frac{U^{\frac{\varepsilon}{2} \beta_{4}}}{2}}\left(\frac{1}{2}|\nabla f|^{2}-W(f)\right) \leq \varepsilon^{1-\beta_{1}}
$$

for all sufficiently small $\varepsilon>0$. We may take the center of $U_{\frac{\varepsilon}{2}-\beta_{4}}\left(=U_{\frac{\varepsilon-\beta_{4}}{2}}(x)\right)$ to be any $x \in U_{s \varepsilon^{-1}}$ so that we have the estimate on $U_{s \varepsilon^{-1}}$. By (3.61), (3.74) and

$$
\sup |W(f)-W(u)| \leq \sup \left|W^{\prime}\right| \sup |u-f| \leq c_{18} \varepsilon^{\beta_{5}},
$$

we may also replace $f$ by $u$ in (3.74) by choosing a larger $0<\beta_{1}<1$ if necessary. This proves the desired estimate.

\section{Rectifiability and integrality of the limit varifold}

In this section, we recover the index $i$ and assume that $\left\{u_{i}\right\}_{i=1}^{\infty},\left\{v_{i}\right\}_{i=1}^{\infty}$ and $\left\{\varepsilon_{i}\right\}_{i=1}^{n}$ satisfy (2.1)-(2.5). Define $\mu_{i}$ and $V_{i}$ as in (2.7) and (2.8). By the standard weak compactness theorem of Radon measures, there exists a subsequence (denoted by the same index) and a Radon measure $\mu$ and a varifold $V$ such that

$$
\mu_{i} \rightarrow \mu, \quad V_{i} \rightarrow V
$$

Lemma 4.1 For $x \in \operatorname{spt} \mu$, there exists a subsequence $x_{i} \in U_{1}$ such that $u_{i}\left(x_{i}\right) \in[-\alpha, \alpha]$ and $\lim _{i \rightarrow \infty} x_{i}=x$.

Proof We prove this by contradiction and assume that there exists some $r>0$ such that $\left|u_{i}\right| \geq \alpha$ on $U_{r}(x)$ for all large $i$. Without loss of generality, assume $u_{i} \geq \alpha$ on $U_{r}(x)$. Then we repeat the same argument leading to (3.30) with $\phi$ there replaced by $C_{c}^{1}\left(U_{r}(x)\right)$. The argument shows that $\lim _{i \rightarrow \infty} \int \frac{\varepsilon_{i}}{2}\left|\nabla u_{i}\right|^{2} \phi^{2}=0$. Next, multiplying $u_{i}-1$ to the equation (2.1) and using $W^{\prime}\left(u_{i}\right)\left(u_{i}-1\right) \geq \frac{\kappa}{2}\left(u_{i}-1\right)^{2}$, we obtain

$$
\begin{aligned}
\int \frac{W}{\varepsilon_{i}} \phi^{2} & \leq c(W) \int \frac{\left(u_{i}-1\right)^{2}}{\varepsilon_{i}} \phi^{2} \leq \frac{2 c(W)}{\kappa} \int \frac{W^{\prime}\left(u_{i}\right)\left(u_{i}-1\right)}{\varepsilon_{i}} \phi^{2} \\
& =\frac{2 c(W)}{\kappa} \int\left(\varepsilon_{i} \Delta u_{i}\left(u_{i}-1\right)+\varepsilon_{i}\left(v_{i} \cdot \nabla u_{i}\right)\left(u_{i}-1\right)\right) \phi^{2} .
\end{aligned}
$$

By integration by parts and (2.5), the right-hand side of (4.1) converges to 0 . This shows that $\mu\left(U_{r}(x)\right)=0$ and contradicts $x \in \operatorname{spt} \mu$. 
Theorem 4.2 There exist constants $0<D_{1} \leq D_{2}<\infty$ which depend only on $c_{0}, \lambda_{0}, n, p, W, E_{0}$ and s such that, for $x \in \operatorname{spt} \mu \cap U_{s}$ and $B_{r}(x) \subset U_{s}$, we have

$$
D_{1} r^{n-1} \leq \mu\left(B_{r}(x)\right) \leq D_{2} r^{n-1} .
$$

Proof This follows immediately from Theorem 3.8, 3.10 and Lemma 4.1.

For the subsequent use, define

$$
\xi_{i}:=\frac{\varepsilon_{i}\left|\nabla u_{i}\right|^{2}}{2}-\frac{W\left(u_{i}\right)}{\varepsilon_{i}} .
$$

Once we have the monotonicity formula (3.55), we may prove the following "equi-partition of energy" by the same proof as in [22, Proposition 4.3]:

Theorem $4.3 \xi_{i}, \frac{\varepsilon_{i}}{2}\left|\nabla u_{i}\right|^{2}-\left|\nabla w_{i}\right|$ and $\frac{W\left(u_{i}\right)}{\varepsilon_{i}}-\left|\nabla w_{i}\right|$ all converge to zero in $L_{\text {loc }}^{1}\left(U_{1}\right)$.

Proof of Theorem 2.1 Recall that $\left\|V_{i}\right\|=\mu_{i}\left\llcorner_{\left\{\left|\nabla u_{i}\right| \neq 0\right\}}\right.$. Since

$$
\sigma \mu_{i}\left\llcorner_{\left\{\left|\nabla u_{i}\right|=0\right\}} \leq\left|\xi_{i}\right| d \mathcal{L}^{n} \rightarrow 0\right.
$$

by Theorem 4.3, $\mu_{i}\left\llcorner_{\left\{\left|\nabla u_{i}\right| \neq 0\right\}}\right.$ converges to $\mu$. We also know that $\left\|V_{i}\right\|$ converges to $\|V\|$ by definition, thus we have $\|V\|=\mu$. This proves (1). The claims (2) and (3) follows from Theorem 3.8, 3.10 and Lemma 4.1 (see also [22, Proposition 4.2]). Next, by (2.9), (3.18) and Theorem 4.3,

$$
\begin{aligned}
\delta V_{i}(g)= & \int_{\left\{\left|\nabla u_{i}\right| \neq 0\right\}} \operatorname{div} g d \mu_{i} \\
& -\frac{1}{\sigma} \int_{\left\{\left|\nabla u_{i}\right| \neq 0\right\}} \nabla g \cdot\left(\frac{\nabla u_{i}}{\left|\nabla u_{i}\right|} \otimes \frac{\nabla u_{i}}{\left|\nabla u_{i}\right|}\right)\left(\varepsilon_{i}\left|\nabla u_{i}\right|^{2}-\xi_{i}\right) \\
= & -\frac{1}{\sigma} \int \varepsilon_{i}\left(v_{i} \cdot \nabla u_{i}\right)\left(\nabla u_{i} \cdot g\right)+o(1)
\end{aligned}
$$

for $g \in C_{c}^{1}\left(U_{1} ; \mathbb{R}^{n}\right)$, where $\lim _{i \rightarrow \infty} o(1)=0$. By Theorem 3.8 and spt $g \subset U_{s}$ for some $0<s<1$, we have a uniform bound on $E(r, x)$ (corresponding to $u_{i}$ ) for $B_{r}(x) \subset U_{s}$. Hence, by Theorem 3.7, we have

$$
\begin{aligned}
& \int \varepsilon_{i}\left(\left(v_{i}-v\right) \cdot \nabla u_{i}\right)\left(\nabla u_{i} \cdot g\right) \leq c\left(\int\left|v_{i}-v\right|^{p}|g|^{p} \varepsilon_{i}\left|\nabla u_{i}\right|^{2}\right)^{\frac{1}{p}} \\
& \leq c\left(\int\left|\nabla\left(\left|v_{i}-v\right|^{p}|g|^{p}\right)\right|\right)^{\frac{1}{p}} \\
& \quad \leq c\left(\left\|\nabla v_{i}-\nabla v\right\|_{L^{p}}\left\|v_{i}-v\right\|_{L^{p}}^{p-1}+\left\|v_{i}-v\right\|_{L^{p}}^{p}\right)^{\frac{1}{p}}
\end{aligned}
$$

where the integrations are over spt $g$. The above converges to 0 since we may choose a further subsequence of $v_{i}$ which converges to $v$ strongly in $L_{l o c}^{p}$. Thus in the right-hand side of (4.3), we may replace $v_{i}$ by $v$. Let $\epsilon>0$ be arbitrary and let $\tilde{v}$ be a smooth vector field such that $\|v-\tilde{v}\|_{W^{1, p}\left(U_{s}\right)}<\epsilon$. By the varifold convergence $V_{i} \rightarrow V$, we have

$$
\begin{aligned}
\frac{1}{\sigma} \int \varepsilon_{i}\left(\tilde{v} \cdot \nabla u_{i}\right)\left(\nabla u_{i} \cdot g\right) & =\int S^{\perp}(\tilde{v}) \cdot g d V_{i}(x, S)+o(1) \\
& \rightarrow \int S^{\perp}(\tilde{v}) \cdot g d V(x, S) .
\end{aligned}
$$


We may arbitrarily approximate the quantities in (4.5) by $v$ by the same argument in (4.4), hence by (4.3)-(4.5), we obtain

$$
\delta V(g)=-\int S^{\perp}(v) \cdot g d V(x, S) .
$$

Hence, $\|\delta V\|$ is a Radon measure on $U_{1}$. By (4.2) and Allard's rectifiability theorem [1, 5.5.(1)], $V$ is rectifiable. Since $V$ is rectifiable, there exist an $\mathcal{H}^{n-1}$ measurable and countably $n-1$-rectifiable set $\Gamma$ such that

$$
\int S^{\perp}(v) \cdot g d V(x, S)=-\int\left(T_{x} \Gamma\right)^{\perp}(v(x)) \cdot g(x) d\|V\|(x) .
$$

The set $\Gamma$ is the measure-theoretic support of $\|V\|$ and $T_{x} \Gamma$ is the approximate tangent space of $\Gamma$ which exists for $\mathcal{H}^{n-1}$ a.e. on $\Gamma$. Next from (4.6), $\|\delta V\|$ is absolutely continuous with respect to $\|V\|$ and the generalized mean curvature $H_{V}$ exists. By (4.6) and (4.7), we have $H_{V}(x)=\left(T_{x} \Gamma\right)^{\perp}(v(x))$ holds for $\|V\|$ a.e. for $x$. This proves (5), except that we do not yet take $\Gamma=\operatorname{spt}\|V\|$. The proof of (4) is the same as [25] for the following reason. We may set $f=\varepsilon \nabla u \cdot v$ in [25] and we have $\|\varepsilon \nabla u \cdot v\|_{L^{\frac{n p}{n-p}}\left(U_{s}\right)} \leq c_{1} \lambda_{0}$ due to Lemma 3.1. In the proof, as long as we have the monotonicity formula (3.55) and the estimate Lemma 3.4, all the argument goes through. The point is that we do not need to take a derivative of $f$ for the proof of integrality and we only need the control of $L^{\frac{n p}{n-p}}$ norm as well as the estimate (3.2). See the comment in the proof of [22, Proposition 4.8] where it is explained that (3.2) is necessary. We should also point out that the $L^{n}$ control of $\nabla f$ is not needed in the proof. Finally, by arguing as in (4.4) and the Hölder inequality, we have

$$
\int_{U_{s}} \phi^{\frac{p(n-1)}{n-p}} d\|V\| \leq c\|\nabla \phi\|_{L^{p}}\|\phi\|_{L^{n p /(n-p)}}^{n(p-1) /(n-p)}
$$

for any function $\phi \in C_{c}^{1}\left(U_{s} ; \mathbb{R}^{+}\right)$and we have the same inequality for $v \in W^{1, p}\left(U_{1}\right)$ by the density argument. Thus we have (6). By the well-known property of varifold having the generalized mean curvature in $L^{q}$ with $q>n-1$ (see [19, 17.9(1)]), spt $\|V\|$ coincide with the measure-theoretic support $\Gamma$, so that we have (5) with $\Gamma=\operatorname{spt}\|V\|$. This concludes the proof of Theorem 2.1.

\section{Concluding remarks}

In [11,21], we studied the singular perturbation problem for

$$
\partial_{t} u_{\varepsilon}+v_{\varepsilon} \cdot \nabla u_{\varepsilon}=\Delta u_{\varepsilon}-\frac{W^{\prime}\left(u_{\varepsilon}\right)}{\varepsilon^{2}}
$$

and proved that the time-parametrized family of limit varifolds satisfies the motion law of "normal velocity $=$ mean curvavture vector $+v^{\perp}$ " in a weak formulation (see [9,23] for the case of $v_{\varepsilon}=0$ ). In these works, we assumed that the prescribed initial data satisfies a boundedness of the upper density ratio. Part of the difficulty was to show that the upper density ratio bound can be controlled locally in time and uniformly with respect to $\varepsilon$. For the equilibrium problem, it is certainly not natural to assume such an upper density ratio estimate. It is interesting to see if one can drop the upper density ratio assumption for the initial data in the proof of $[11,21]$.

The vectorial prescribed mean curvature problem as in Theorem 2.2 seems, as far as we know, little studied so far. Traditionally, the prescription is the scalar version, i.e. given a 
scalar function (or constant) $f$, one looks for a hypersurface satisfying $H \cdot v=f$, where $v$ is the normal unit vector. The vectorial version is physically natural from the view point of force balance, in that the problem seeks the equality between the surface tension force and an external force acting on the surface. It must be said that the prescribed vector field in Theorem 2.2 is the gradient of a potential $\rho$, and not a general vector field. This is rather restrictive for applications and it is interesting to know if there can be a remedy for generalizations. If there may not exist a variational framework such as the min-max method to find solutions of (1.2), it should be still useful to have this diffused interface approach since the functional is well-behaved functional-analytically. As a further question, it is also interesting to investigate the asymptotic behavior of stable critical points of $F_{\varepsilon}$ in the proof of Theorem 2.2, since we have a very successful analogy in $[24,26]$. In this direction, we mention that a construction of prescribed scalar mean curvature hypersurfaces along the lines suggested here has been announced recently [3] (see also [2]).

Open Access This article is licensed under a Creative Commons Attribution 4.0 International License, which permits use, sharing, adaptation, distribution and reproduction in any medium or format, as long as you give appropriate credit to the original author(s) and the source, provide a link to the Creative Commons licence, and indicate if changes were made. The images or other third party material in this article are included in the article's Creative Commons licence, unless indicated otherwise in a credit line to the material. If material is not included in the article's Creative Commons licence and your intended use is not permitted by statutory regulation or exceeds the permitted use, you will need to obtain permission directly from the copyright holder. To view a copy of this licence, visit http://creativecommons.org/licenses/by/4.0/.

\section{References}

1. Allard, W.: On the first variation of a varifold. Ann. Math. (2) 95, 417-491 (1972)

2. Bellettini, C., Chodosh, O., Wickramasekera, N.: Curvature estimates and sheeting theorems for weakly stable CMC hypersurfaces. Adv. Math. 352, 133-157 (2019)

3. Bellettini, C., Wickramasekera, N.: Stable CMC integral varifolds of codimension 1: regularity and compactness. arXiv: 1802.00377

4. Cahn, J.W., Hilliard, J.E.: Free energy of a nonuniform system I. Inter facial free energy. J. Chem. Phys. 28, 258-267 (1958)

5. Gilbarg, D., Trudinger, N.: Elliptic Partial Differential Equations of Second Order, 2nd edn. Springer, Berlin (1983)

6. Guaraco, M.A.M.: Min-max for phase transitions and the existence of embedded minimal hypersurfaces. J. Differ. Geom. 108(1), 91-133 (2018)

7. Gurtin, M.E.: Some results and conjectures in the gradient theory of phase transitions. In: Metastability and Incompletely Posed Problems (Minneapolis, Minn., 1985). IMA Vol. Math. Appl., vol. 3, pp. 135-146. Springer, New York (1987)

8. Hutchinson, J.E., Tonegawa, Y.: Convergence of phase interfaces in the van der Waals-Cahn-Hilliard theory. Calc. Var. Partial Differ. Equ. 10(1), 49-84 (2000)

9. Ilmanen, T.: Convergence of the Allen-Cahn equation to Brakke's motion by mean curvature. J. Differ. Geom. 38(2), 417-461 (1993)

10. Kohn, R.V., Sternberg, P.: Local minimisers and singular perturbations. Proc. R. Soc. Edinb. Sect. A 111(1-2), 69-84 (1989)

11. Liu, C., Sato, N., Tonegawa, Y.: Two-phase flow problem coupled with mean curvature flow. Interfaces Free Bound. 14(2), 185-203 (2012)

12. Luckhaus, S., Modica, L.: The Gibbs-Thompson relation within the gradient theory of phase transitions. Arch. Ration. Mech. Anal. 107(1), 71-83 (1989)

13. Modica, L.: The gradient theory of phase transitions and the minimal interface criterion. Arch. Ration. Mech. Anal. 98(2), 123-142 (1987)

14. Modica, L., Mortola, S.: Un esempio di $\Gamma$-convergenza. Boll. Un. Mat. Ital. 14-B, 285-299 (1977)

15. Nagase, Y., Tonegawa, Y.: A singular perturbation problem with integral curvature bound. Hiroshima Math. J. 37(3), 455-489 (2007) 
16. Pitts, J.T.: Existence and Regularity of Minimal Surfaces on Riemannian, Manifolds Mathematical Notes, vol. 27. Princeton University Press, Princeton (1981)

17. Röger, M., Schätzle, R.: On a modified conjecture of De Giorgi. Math. Z. 254(4), 675-714 (2006)

18. Röger, M., Tonegawa, Y.: Convergence of phase-field approximations to the Gibbs-Thomson law. Calc. Var. Partial Differ. Equ. 32(1), 111-136 (2008)

19. Simon, L.: Lectures on geometric measure theory. In: Proceedings of the Centre for Mathematical Analysis, Australian National University, vol. 3 (1983)

20. Sternberg, P.: The effect of a singular perturbation on nonconvex variational problems. Arch. Ration. Mech. Anal. 101(3), 209-260 (1988)

21. Takasao, K., Tonegawa, Y.: Existence and regularity of mean curvature flow with transport term in higher dimensions. Math. Ann. 364, 857-935 (2016)

22. Tonegawa, Y.: Phase field model with a variable chemical potential. Proc. R. Soc. Edinb. Sect. A 132(4), 993-1019 (2002)

23. Tonegawa, Y.: Integrality of varifolds in the singular limit of reaction-diffusion equations. Hiroshima Math. J. 33(3), 323-341 (2003)

24. Tonegawa, Y.: On stable critical points for a singular perturbation problem. Commun. Anal. Geom. 13(2), 439-459 (2005)

25. Tonegawa, Y.: A diffused interface whose chemical potential lies in Sobolev spaces. Ann. Scuola Norm. Sup. Pisa Cl. Sci. (5) 4, 487-510 (2005)

26. Tonegawa, Y., Wickramasekera, N.: Stable phase interfaces in the van der Waals-Cahn-Hilliard theory. J. Reine Angew. Math. 668, 191-210 (2012)

27. Wickramasekera, N.: A general regularity theory for stable codimension 1 integral varifolds. Ann. Math. 179(3), 843-1007 (2014)

28. Zhou, X., Zhu, J.J.: Min-max theory for constant mean curvature hypersurfaces. Invent. Math. 218(2), 441-490 (2019)

29. Zhou, X., Zhu, J.J.: Existence of hypersurfaces with prescribed mean curvature I-generic min-max. Camb. J. Math. 8(2), 311-362 (2020)

30. Ziemer, W.P.: Weakly Differentiable Functions. Springer, Berlin (1989)

Publisher's Note Springer Nature remains neutral with regard to jurisdictional claims in published maps and institutional affiliations. 